\title{
Der Reichsritter Wolf Heinrich von Ega zu Ober- und Unterschüpf
}

\section{Ungelöste Fragen zwischen Vorarlberg und Schüpfergrund}

\author{
von Helmut Neumaier
}

Wer immer sich mit der fränkischen Reichsritterschaft beschäftigt, wird Johann Gottfried Biedermann (1705-1766) und das, was von seinem Lebenswerk geblieben ist, nicht außer Acht lassen dürfen. In der, Vorrede‘ zum , Geschlechts-Register [...] Orts Gebürg' attestierte er seinen adligen Gewährsmännern denn auch höchstes Bemühen, viele bis anhero verborgen gelegene Warheiten aus dem Schimmel und Moder der alten fast dunckel gewordenen Urkunden, hervor suchen zu lassen. ${ }^{1}$ Das ist einerseits Topik, doch erlaubt das auch einen bemerkenswerten Einblick in das Archivwesen so manches Edelmanns. Zugleich stellt diese Bemerkung Biedermanns genealogischen Fähigkeiten ein gutes Zeugnis aus. Selbstredend ist das Urteil Franz Xaver von Wegeles nicht schönzureden, wonach nicht wenige der Angaben, vor allem zu den älteren Zeiten, mit höchster Vorsicht zu bewerten sind. ${ }^{2}$ Dies gilt vor allem für die Tatsache, dass nicht wenige seiner adligen Gewährsmänner ihrer Familie vor allem mittels des Rixnerschen Turnierbuchs, ${ }^{3}$ und das im besten Glauben, ein besonders hohes Alter zu verschaffen glaubten oder es zumindest wünschten; und Biedermann ist ihnen darin gefolgt.

Mit bemerkenswerter Akribie hat Biedermann Verzeichnisse der Mitgliedsfamilien der sechs fränkischen Ritterorte/Kantone erstellt. Bei den ,Geschlechts-Registern' des Orts/Kantons Odenwald, getrennt nach erloschenen und zu seiner Zeit noch blühenden Häusern, ist man selbst für erstere von der Vollständigkeit überrascht. Grenzt man auf das durch eine besonders hohe Adelsdichte gekennzeichnete Bauland, den Raum zwischen Hinterem Odenwald, Tauber, Jagst und Neckar, ein, vermisst man allerdings im Verzeichnis der erloschenen Familien drei Namen: Leyen (Layen), Dienheim und Ega. Bei den beiden ersten handelt

1 Johann Gottfried Biedermann: Geschlechts-Register Der Reichs-Frey-unmittelbaren Ritterschafft Landes zu Francken Löblichen Orts-Gebürg. Bamberg 1747 (ND Neustadt/Aisch 1984).

2 Franz Xaver von Wegele: Biedermann, Johann Gottfried. In: ADB 3 (1876), S. 793 f.

3 Klaus Arnold: Der fränkische Adel, die ,Turnierchronik“ des Jürg Rugen (1494) und das Turnierbuch des Georg Rixner (1530). In: Erich Schneider (Hg.): Nachdenken über fränkische Geschichte (Veröffentlichungen der Gesellschaft für fränkische Geschichte, IX/50). Neustadt/Aisch 2005, S. 129-153. 


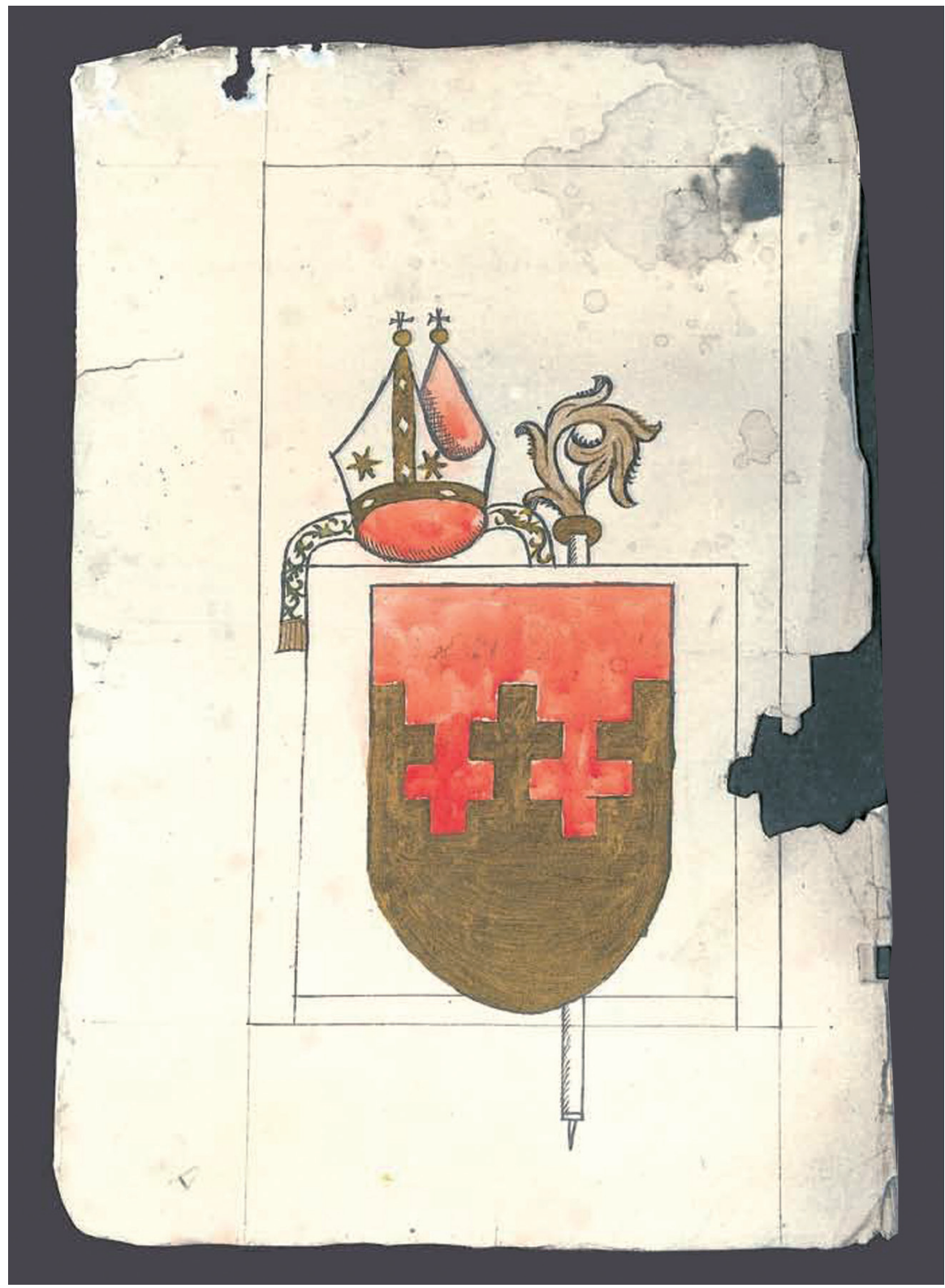

Wappen des Murrhardter Abtes Wilhelm Egen (1469-1486), Vorlage und Aufnahme: HStA Stuttgart A 508, Bü 1. Alle Rechte vorbehalten. 
es sich um wohlbekannte Familien aus der Ritterschaft am Rheinstrom, wogegen man den Namen Ega dort vergeblich sucht. Dafür verzeichnet das Siebmachersche Wappenbuch beim schwäbischen Adel eben diesen Namen und das zugehörige Wappen: ${ }^{4}$ gevierter Schild, Feld 1 und 4 Zinnenschnitt in Rot und Silber, 2 und 3 Rüde silber im roten Feld. Deshalb wird man nicht ohne Erstaunen in der Einladungsliste des Orts/Kantons Odenwald zum Mergentheimer Rittertag am 4. Januar 1596 einen Wolf Heinrich von Ega entdecken. ${ }^{5}$ Um ihn, der sich $z u$ Obern- und Unternschüpf nannte, geht es im Folgenden.

\section{Der Schüpfergrund auf dem Weg zur Vielherrigkeit}

Um ermessen zu können, in welch kompliziertes Umfeld dieser Wolf Heinrich von Ega eintrat, ist die besondere Herrschaftsstruktur des Schüpfergrundes zu skizzieren. Ihr Entstehen vollzog sich in zwei zeitlichen Stufen: 1572 Tod des Albrecht von Rosenberg zu Schüpf, 1632 Erlöschen des Gesamthauses derer von Rosenberg. ${ }^{6}$

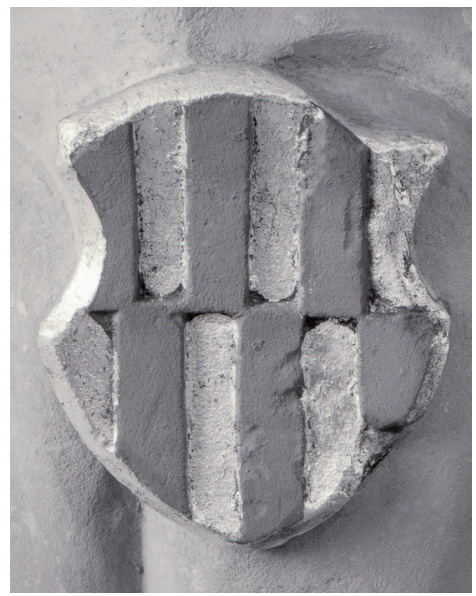

Wappen Rosenberg an der Kanzel der Kirche in Unterschüpf (Foto: Neumaier).

4 Johann Siebmacher: New Wappenbuch: Darinnen deß H. Röm. Reichs Teutscher Nation hoher Potentaten, Fürsten, Herren, und Adelspersonen auch anderer Ständt und Stätte Wappen. Bd. 1, fol. 121, mittlere Reihe, 3. von links; Abbildung nach der Vorlage von 1564 bei Otto von Alberti: Württembergisches Adels- und Wappenbuch. Bd. 1. Stuttgart 1889, S. 149 f. Geht man von Tettnang, dem Ansitz des Hans Christoph von Ega aus, war dieser Mitglied des Kantons Hegau-Allgäu-Bodensee der schwäbischen Reichsritterschaft.

5 StAL JL 425 Sammlung Breitenbach, Bd. IX Nr. 31.

6 Helmut Neumaier: Als sterblicher Mensch dem Todt unterworffen - Das Testament des Albrecht Christoph von Rosenberg aus dem Jahre 1630. In: Wertheimer Jahrbuch 1991/92 (1992), S. 81-95. 
Albrecht von Rosenberg hatte es verstanden, aus den Dörfern des Schüpfergrundes - Unter- und Oberschüpf, Uiffingen, Lengenrieden, Kupprichhausen, Schweigern - sowie Dainbach, Buch am Ahorn, Brehmen und Sachsenflur eine geschlossene Flächenherrschaft aufzubauen. ${ }^{7}$ Da der damals weithin berühmte Ritter sich auch die kirchlichen Rechte zu sichern wusste, findet die Schüpfer Herrschaft beim reichsritterschaftlichen Adel kaum etwas Vergleichbares. Auf den ersten Blick einem kleinen Territorium gleichkommend, ist jedoch auch hier die strukturelle Schwäche der allermeisten Adelsherrschaften festzustellen. Sie bestand weniger im Fehlen der Zentrechte, die dem Erzstift Mainz zukamen, die der Ritter aber zu entschärfen gewusst hatte, als vielmehr in der Lehnbindung an Mainz und das Hochstift Würzburg, daneben auch an die Grafschaft Hohenlohe und andere. Der Allodialbesitz trat dahinter weit zurück.

Mit dem Tod des Ritters zerfiel die geschlossene Herrschaft Schüpf. Leider ist sein Testament nicht erhalten, doch kann es in großen Zügen rekonstruiert werden: Die Mannlehen fielen an seine Vettern, während er die Erblehen den Cognaten - den Leyen und den Stetten zu Kocherstetten - zudachte. Es dürfte kaum zu gewagt sein, aus dieser Tatsache auf Spannungen zwischen Ritter Albrecht und den anderen Familienzweigen zu schließen, doch fehlen Quellenzeugnisse. Mit der testamentarischen Verfügung, seine Herrschaft Schüpf unter Agnaten und Cognaten aufzuteilen, hatte Ritter Albrecht von Rosenberg das Problem geschaffen, wie dieses so entstandene politische Gebilde verfassungsrechtlich definiert werden kann.

Die Formulierung „Herrschaft Schüpf“ weicht der Frage aus und kann wirkliche Geltung eigentlich nur für die Zeit des Ritters Albrecht beanspruchen. Sie trifft insofern zwar den Kern, als Herrschafts- und Besitzrechte in den Dörfern des Schüpfergrundes mehr als einem Herrn zukamen, sie besagt aber noch nichts über die rechtliche Qualität. Bekanntlich tat sich schon die Reichspublizistik mit der juristischen Systematisierung von Herrschaftsgemeinschaften alles andere als leicht. ${ }^{8}$ Für Jakob Ernst Leutwein (1684-1763), Pfarrer und Historiograph des Schüpfergrundes, war die Sache eindeutig, wenn er von den Rosenberg, Stetten und Dienheim als „Gan-Erben“ spricht. ${ }^{9}$ Wir wissen nicht, ob er die zeitgenössische staatsrechtliche Literatur kannte. Den gebildeten Zeitgenossen und rechtlich Interessierten, zu denen selbstverständlich auch er zählte, dürften die Begriffe Ganerben und Ganerbschaft geläufig gewesen sein. Einige Zeit nach

7 Helmut Neumaier: Albrecht von Rosenberg. Ein außergewöhnliches Adelsleben unter drei habsburgischen Kaisern. Münster 2011, S. 179-212.

8 Dazu Alexander Jendorff: Condominium. Typen, Funktionsweisen und Entwicklungspotenziale von Herrschaftsgemeinschaften in Alteuropa (Veröffentlichungen der Historischen Kommission für Hessen 72). Marburg 2010, S. 69-105.

9 Beispielsweise Jakob Ernst Leutwein: Schüpfer Kirchenhistorie, Bd. 3: Epitomae Historiae Schupfiensis Politicae (handschr., beendet 1761), S. 164: Nachdeme Aegidius Reinhard von Dienheim [...]d(e)n 13. Dec. 1589 gestorben, bliebe deßen Gemahlin Rufina von Leyen, durch welche er Ganerbe Schüpfer Grunds worden, 5 Jahr lang im Wittwenstand. - Verf. muss gestehen, dass er in früheren Publikationen zum Schüpfergrund ebenfalls von Ganerbschaft und Ganerben ausging. 


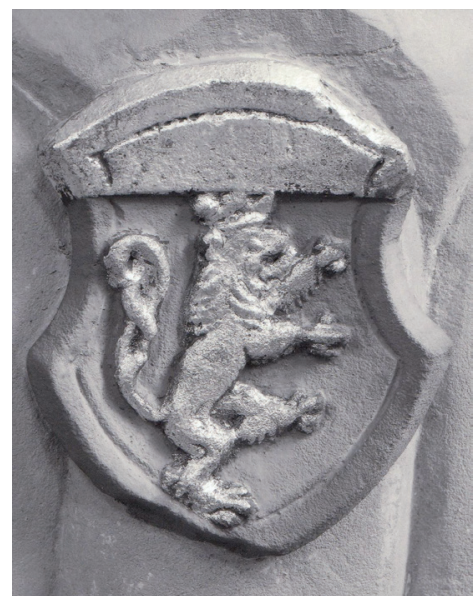

Wappen Dienheim an der Kanzel der Kirche in Unterschüpf (Foto: Neumaier).

Leutwein hat der berühmte Verfassungsrechtler Johann Jakob Moser die Feststellung getroffen, die Verfassung der Ganerbschaften seynd verschiden. ${ }^{10}$ Hat Moser dieses Rechtsinstrument in ein System gebracht, ist sie im landläufigen Verständnis wohl recht undifferenziert gebraucht worden.

Vergleicht man Schüpf mit tatsächlichen Ganerbschaften - um nur einige wenige wie die Ganerbenburg Rothenberg ${ }^{11}$, die Reichsburg Friedberg ${ }^{12}$ und die Stadt Künzelsau im Kocherta ${ }^{13}{ }^{13}$ u nennen -, wird der Unterschied evident. Bei ihnen liegen gemeinschaftliche Besitz- und Herrschaftsrechte vor, was für Schüpf nicht zutrifft. Während bei allen Unterschieden im Detail dort die Ganerben ihre Rechte gemeinsam ausübten, besaßen im Schüpfergrund drei Familien separate Besitz- und Vogteirechte. Von einem Kondominat unterscheidet es sich durch die Tatsache, dass es sich bei ihnen nicht um Reichsunmittelbare handelte, ${ }^{14}$ sondern um Angehörige des Corpus equestre und Lehenträger des Erzstifts Mainz, daneben auch der Grafschaft Hohenlohe. Damit gelangt man zu dem Begriff der

10 Johann Jakob Moser: Teutsches Staatsrecht. Bd. 3: Von denen kayserlichen Regierungs-Rechten und Pflichten. Zweyter Theil. Frankfurt/Main 1773, S. 150 f.; allgemein Werner Ogris: Stichwort Ganerbschaft. In: Handwörterbuch zur deutschen Rechtsgeschichte. Bd. 1. Berlin ${ }^{2} 2008$, S. 19281930.

11 Klaus Rupprecht: Ritterschaftliche Herrschaftswahrung in Franken (Veröffentlichungen der Gesellschaft für Fränkische Geschichte IX/42). Neustadt/Aisch 1994, S. 377-383.

12 Klaus-Dieter Rack: Die Burg Friedberg im Alten Reich. Studien zu ihrer Verfassungs- und Sozialgeschichte zwischen dem 15. und 19. Jahrhundert. Darmstadt, Marburg 1988.

13 Werner Nowak: Die Ganerbschaft Künzelsau. Diss. phil. Tübingen 1966; Günter Christ: Erzstift und Territorium Mainz. In: Friedhelm Jürgensmeier (Hg.): Handbuch der Mainzer Kirchengeschichte. Bd. 2, Würzburg 1994, S. 17-444, hier S. $181 \mathrm{f}$.

14 Dietmar Willoweit: Stichwort Kondominat. In: Handwörterbuch zur deutschen Rechtsgeschichte. Bd. 2. Berlin 2012, Sp. 212-214. 
„vielherrigen Dörfer“, dem „Nebeneinander verschiedener territorialer Gewalten über einzelne Hofstätten und Gerechtsame am selben Ort" ${ }^{\text {. }}{ }^{5}$

Der Schüpfer Befund entzieht sich jedoch der klaren Definition, denn innerhalb der Vielherrigkeit bestanden sehr wohl Ganerbenrechte. Zumindest ist dies für die Brüder von Rosenberg nachweisbar, die am 2. August 1586 eine Nutzteilung (Mutschierung) vornahmen. ${ }^{16}$ Wie dem auch sei, der Schüpfergrund war zu einem höchst komplexen politischen Gebilde geworden. Nicht zu übersehen ist allerdings die Dominanz der drei Brüder der letzten Generation des Hauses Rosenberg - Konrad XIII. (gest. 1596), Georg Sigmund (gest. 1630) und Albrecht Christoph (gest. 1632). Um der Einfachheit willen - darauf sei an dieser Stelle hingewiesen - wird im Folgenden der Terminus Ganerben bzw. Ganerbschaft beibehalten.

\section{Die von Dienheim - die dritte Familie}

Um das Folgende verstehen zu können, muss hier etwas weiter ausgeholt und zur ersten der beiden Stufen zurückgekehrt werden. Es bleibt nicht erspart - um mit Thomas Manns Hanno Buddenbrook zu sprechen, - sich ins „genealogische Gewimmel“" zu stürzen.

Die Mutter des Ritters Albrecht von Rosenberg hatte nach dem Tod des Gatten den pfalz-simmerschen Hofmeister und Amtmann zu Winterberg Peter von Leyen (gest. 1552) geheiratet. Dieser zweiten Ehe entstammten zwei Kinder, nämlich Margarethe (urk. 1549, gest. 5. November 1589) und Eberhard (urk. 1532, gest. 21. Dezember 1572). Von ihnen gingen neben den Rosenberg zwei weitere Anteilseigner der Herrschaft Schüpf aus. Margarethe ehelichte Eberhard von Stetten zu Kocherstetten. Eberhard von Leyen hinterließ eine Tochter, die den Namen nach Ritter Albrechts zweiter Gattin Ruf(f)ina führte. Man muss sich bewusst sein, dass man sich genealogisch auf unsicherem Terrain bewegt, sodass Korrekturen nicht auszuschließen sind. ${ }^{17}$

Mehr als die Tatsache, dass bereits vor dem Tod des Ritters Albrecht das Dörfchen Angeltürn sich im Besitz des Albrecht (II.) ${ }^{18}$ von Dienheim zu Dexheim befand, der hier 1564 die Schüpfer Dorfordnung einführte, ${ }^{19}$ ist nicht bekannt. Man darf aber annehmen, dass er die Heiratsverbindung mit den Leyen ange-

15 Ebd., Sp. 2013.

16 Evang. Pfarramt Rosenberg. Sogenanntes „Befehlbuch“, S. 91; Helmut Neumaier: Als sterblicher Mensch dem Todt unterworffen - Das Testament des Albrecht Christoph von Rosenberg aus dem Jahre 1632. In: Wertheimer Jahrbuch 1991/92, S. 81-95, hier S. 85.

17 Zu den Dienheim bisher nur Wigbert G. Faber: Aus der Geschichte von Dienheim. Bd. 3: Die Ritter und Reichsfreiherren von Dienheim. Museum am Siliusstein 2012. Das Schwergewicht liegt so gut wie ganz auf den rheinischen Dienheim, sodass es zwischen ihm und Leutwein (wie Anm. 9) gelegentlich zu Widersprüchen kommt.

18 Die Zählung der Albrechte folgt Faber (wie Anm. 17).

19 Fürstlich-Leiningisches Archiv Amorbach 8-13-6. 


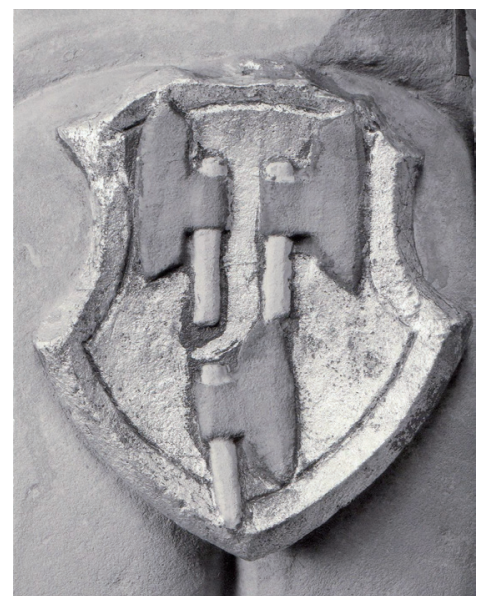

Wappen Kocherstetten an der Kanzel der Kirche in Unterschüpf (Foto: Neumaier).

strebt hat. Als Ruf(f)ina von Leyen im Jahre 1577 Ägidius Reinhard von Dienheim $^{20}$ ehelichte, hatte sich neben den Rosenberg und den Stetten zu Kocherstetten die dritte Ganerbenfamilie formiert.

Mit einiger Irritation vermerkte Leutwein in seinem 1761 beendeten Werk, einer der Dienheim habe sich neben Angertal (Angeltürn) auch zu Rüdelsheim und Hanheim geschrieben. ${ }^{21}$ Was er nicht wissen konnte, dass die Dienheim zu Schüpf aus dem Rheinhessischen stammten und dort noch über Besitzungen, hier also Rüdelsheim und Hahnheim (beide Lkr. Mainz-Bingen), verfügten. Das bedarf noch der genaueren familiengeschichtlichen Untersuchung, doch wird man so viel doch sagen können, dass die Dienheim sich in einen rheinisch-katholischen und einen evangelischen Schüpfer Zweig aufspalteten, wobei letzterer noch über einige Besitzungen bei Oppenheim verfügte. Hier wird die Strategie erkennbar, ein Auseinanderbrechen der Familie unter allen Umständen zu vermeiden. Ganz offensichtlich gab es ein Hausgesetz, wonach die Belehnung aller Zweige ausschließlich dem Ältesten der Gesamtfamilie als Träger zukam. In den Lehenreversen gegenüber dem Erzstift Mainz wird dieses Recht des Familienältesten bei jeder Belehnung ausdrücklich betont. ${ }^{22}$

Der genannte Ägidius Reinhard starb laut der gedruckten Leichenpredigt am 23. Dezember 1589 (a. St.). Dieses Dokument lag Leutwein also noch vor, ist leider aber nicht mehr erhalten. ${ }^{23}$ Der Ehe entsprossen neben einer Tochter drei überle-

20 Den Vornamen Ägidius überliefert nur Leutwein (wie Anm. 9), S. 159, doch wird er hier beibehalten.

21 Ebd., S. 161.

22 StA Würzburg. Mainzer Lehenbuch Nr. 18-19, 21, 24, 28-29 u. 35.

23 Nach dem Belehnungsgesuch an Graf Wolfgang von Hohenlohe am 15. Dezember. 
bende Söhne. ${ }^{24}$ Die Tochter, Margarethe, verheiratet mit Hans Burkhard von Itzstein, starb 1623 im ersten Kindbett. ${ }^{25}$ Von den Söhnen starb Philipp Adam (I.) im Jahre 1605 ohne Nachkommen; sein Besitzanteil wurde am 24. Juni 1606 durch Los unter seinen Brüdern geteilt: Albrecht Nikolaus trat in den Johanniter-, Wilhelm Albrecht in den Deutschorden ein. Hans Reinhard blieb unverheiratet, lebte - wie Leutwein es ausdrückt - in coelibatu und starb am 3. Juni 1640. Wie Leutwein ferner erzählt, sei er im Dreißigjährigen Krieg vielen Gewalttätigkeiten ausgesetzt gewesen und habe durch häufige Plünderungen sein Vermögen verloren. ${ }^{26}$ Mit ihm erlosch der Oberschüpfer Zweig der Dienheim. ${ }^{27}$

Der zweite Sohn des zu Dexheim gesessenen Albrecht (II.) war der namensgleiche Albrecht (III.), der nicht nur innerhalb der Familie, sondern auch beim umwohnenden Adel, insbesondere den anderen Ganerben, für erheblichen Unmut sorgte. Wie dann Wolf Heinrich von Ega am 8. Februar 1613 dem Grafen Georg Friedrich von Hohenlohe berichtete, sei er ebenso leichtlebig gewesen wie sein Vater. Dieser habe seinerzeit aus Speyer eine leichtsinnige Person mitgebracht, einige Zeit mit ihr in Mergentheim gelebt und sie auch geheiratet. ${ }^{28}$ Der Ehe Albrechts (III.) und der Margarethe Ebel entsprossen angeblich neun Kinder, von denen jedoch beim Tod des Vaters im Jahre 1613 nur fünf nachzuweisen sind: ${ }^{29}$ Hans Philipp 23, Albrecht 21, Ebert Georg Friedrich 13, Ruffina 24, Elisabeth 14 Jahre alt.

Auf die Nachricht vom Tod Albrechts (III.) beauftragte Graf Georg Friedrich von Hohenlohe seinen Weikersheimer Vogt Wolf Koler mit Erkundungen. Dessen Bericht vom 28. August 1613 ist höchst aufschlussreich: Von den Söhnen lebte der jüngste, Eberhard (gemeint ist Ebert Georg Friedrich), bei seinem Onkel in Oppenheim, die anderen bei der Mutter im verkauften Unterschüpfer Schloss. Den ältesten, Johann Philipp, nahm der Bischof von Bamberg als Kammerjungen an, hat ihn wegen unzüchtigen, üblen Verhaltens aber balden wieder abgeschafft. Außer den anderen Dienheimern haben die Kinder keine Vormünder. Die benachbarten Edelleute weigern sich aufgrund vieler Schulden, unor-

24 Etwas mehr Klarheit in die Genealogie bringen die Lehenreverse Bd. 24, fol. 126r-v und Bd. 28, 245r-v. Ersterer am 22. Mai 1602 als Träger Philipp Adam für sich, seine Brüder Eberhard, Hans Heinrich und Albrecht, die Söhne des verstorbenen Albrecht; sodann die Söhne ihres verstorbenen Bruders Reinhard, nämlich Albrecht Nikolaus, Wilhelm Albrecht und Hans Reinhard u. a. m. Am 4. Juli 1605 die Brüder Eberhard und Hans Heinrich; sodann die Vettern Hans Dietrich und Hans Eberhard für Reinhards, des Bruders des Eberhard, nachgelassene Söhne Albrecht Nikolaus, Wilhelm Albrecht und Reinhard.

25 Jakob Ernst Leutwein: Schüpfer Kirchenhistorie. Erstes Buch. Sectio I Caput IV, S. 16f.: Leichenpredigt und elegisches Distichon durch Superintendent M. Erhard Happach.

26 Leutwein (wie Anm. 9), S. $161 \mathrm{f}$.

27 Als Hohenlohe 1619 seine Lehnsleute zur Heeresfolge aufmahnte, werden Hans Reinhard vom Oberschüpfer Zweig und vom Unterschüpfer Albrecht Nikolaus, Hans Philipp der Ältere sowie Albrecht genannt; vgl. Leutwein (wie Anm. 9), S. 161.

28 HZAN GA 20 Gem. Lehenarchiv Schublade XXIV Nr. 11 Dienheim.

29 Ebd. 


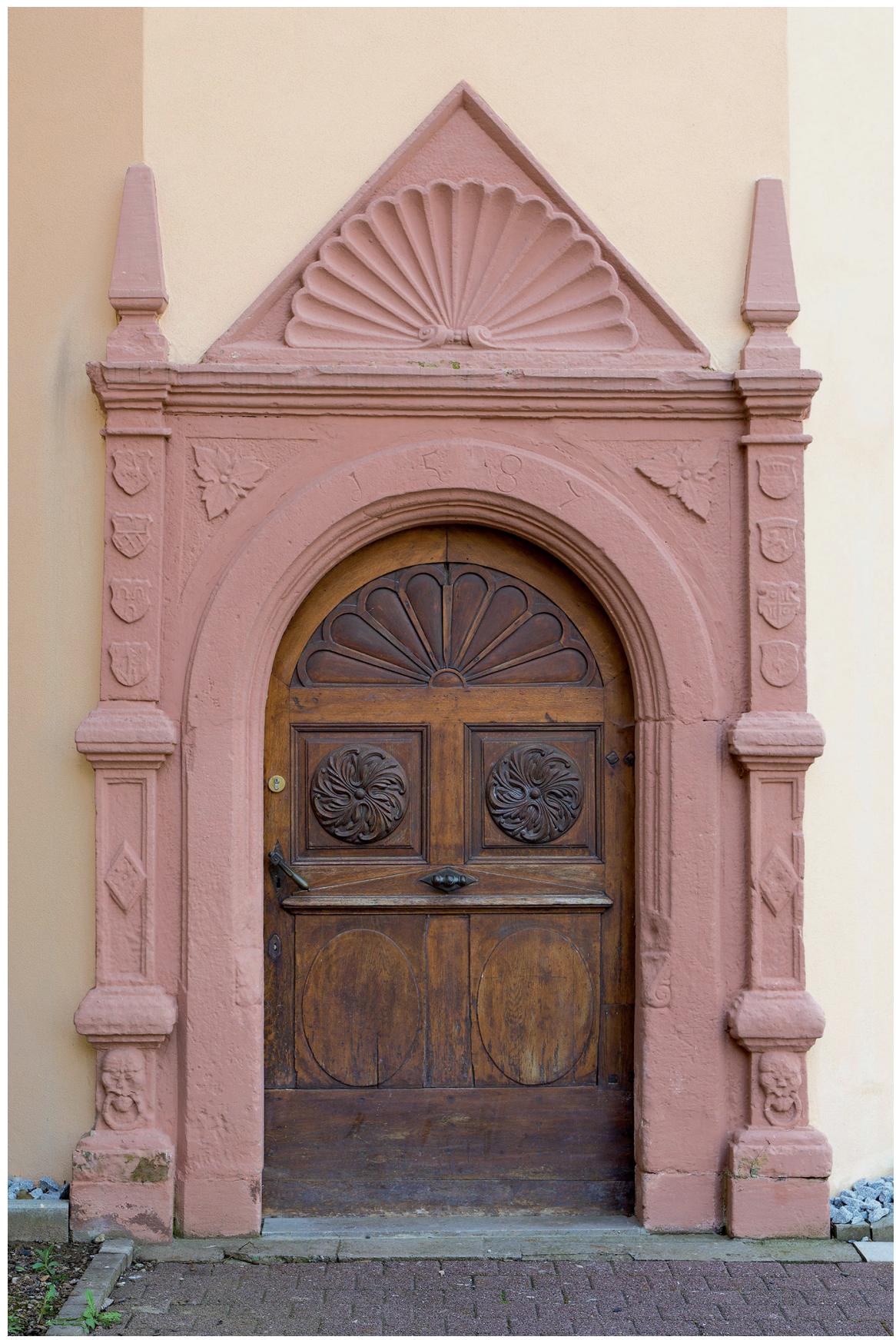

Portal des Schlösschens in Oberschüpf (Foto: Neumaier). 
dentlichen Haushaltens und dass sie, Wittib, nicht edel, die Vormundschaft zu übernehmen. Die Mesalliance war geradezu unverzeihlich. ${ }^{30}$

Mag der gräfliche Vogt die wirtschaftlichen Verhältnisse auch etwas zu drastisch dargestellt haben, das folgende Szenario ist dennoch aufschlussreich genug: Die Witwe und ihr Vater Heinrich Ebel nützten zwar die Güter, aber solche Administration gereicht zu der Kinder äußerstem Nachtheil und Verderben, da gemelter ihr Anherr bißhero die Gültfrüchten und andere Einkommen offt ein ganz Jar eher verkaufft dann dieselbig fellig gewesen unndt nicht den halben Werth daraus erlöst, immassen Freut unndt Vorfreut, das Malter Korn nur pro $2 \mathrm{fl}$ hingeben, sohere nach vor 5 biß in die 7 golten, auch unlangest die künfftige Jarsgefell wider verkaufft und darzu das Gelt mehrertheils für sich allein verzehrt und verspiehlt, wenig ine Haushaltung dargibt, als daß Mutter und Kinder vielmahlen Hunger leiden unndt nicht das tägliche Brodt zu essen haben.

Zur Mesalliance war also noch die Misswirtschaft getreten. Zudem bestanden zwischen den Brüdern Ägidius Reinhard und Albrecht (III.) erhebliche Dissensen, die in gewaltsamer Auseinandersetzung zu eskalieren drohten. Wie der pfälzische Amtmann von Boxberg Friedrich Husman von Namedy seinem Herrn berichtete, sei ersterer dem Bruder mit einer Spießrute gegenüber gestanden.

Die Spannungen drückten sich schon bei der Wahl der Ansitze aus. Ägidius Reinhard hatte sich 1587 in Oberschüpf ein eigenes Schlösschen erbaut, von dem leider nur noch ein stattliches Kellergewölbe und der Treppenturm erhalten sind. ${ }^{31}$ Etwas abschätzig - und das bezog Wolf Heinrich von Ega mit ein - sprachen später die Rosenberg von denen zu Oberschüpf. Dagegen bewohnten Albrecht (III.) und seine Familie das Schlösschen in Unterschüpf. 1610 verkauften sie es um $26000 \mathrm{fl}$ an die Rosenberg, ${ }^{32}$ die es niederreißen ließen, um dafür einen stattlicheren Bau zu errichten. ${ }^{33}$ Anscheinend besaß der Unterschüpf-Angeltürner Familienzweig noch eine Zeit lang hier Wohnrecht. Der Verkaufserlös, den sie von den Rosenbergern erhalten hatten, gab Albrechts (III.) Sohn, dem schon genannten Hans Philipp, die Möglichkeit, in Angeltürn einen bescheidenen Ansitz, das später sogenannte Ficksche Schlösschen, zu errichten.

Verheiratet war er mit Amalia Elisabeth, der Tochter des Christoph Rüdt zu Eubigheim, ${ }^{34}$ was zeigt, dass Konnubium mit dem indigenen Adel inzwischen wieder möglich geworden war. Dieser Ehe entsprossen Johann Philipp der Jüngere und Heinrich Albrecht. Sie verkauften am 6./7. März 1668 Ansitz und Dörfchen

30 Zur Betonung des Geblüts sei hier nur auf H.C. Erik Midelfort: Adeliges Landleben und die Legitimationskrise des deutschen Adels im 16. Jahrhundert. In: Georg Schmidt (Hg.): Stände und Gesellschaft im alten Reich. Stuttgart 1989, S. 245-264, hier S. 261 f. verwiesen.

31 Adolf von Oechelhäuser (Bearb.): Die badischen Kunstdenkmäler. Die Kunstdenkmäler des Amtsbezirks Tauberbischofsheim. Freiburg 1898, S. 214.

32 HZAN Ni 10 B 292.

33 Ebd.

34 Walther Möller: Stammtafeln westdeutscher Adelsgeschlechter. Bd. 3. Darmstadt 1936, Taf. CXXXVI. 
dem pfälzischen Oberamtmann zu Boxberg Georg Wilhelm von Brunn. ${ }^{35}$ Damit - möglicherweise nach einem Zwischenspiel in Merchingen ${ }^{36}$ - verschwanden die Dienheim aus dem fränkischen Raum. In einem Nachtrag überliefert Leutwein, dass die letzte Unterschüpf-Angeltürn-Dienheim-Tochter, Eva Margaretha Katharina, das wenige, was nach Bezahlung der Schulden übrig geblieben war, erbte und sie mit Wolfgang Eberhard Cappler von Oedheim genannt Bautz verheiratet war. $^{37}$

\section{Vom städtischen Patriziat zur Reichsritterschaft: die Egen/Ega}

Den Kern der Reichsritterschaft bildete zweifellos der Burgenadel, wobei nicht jedes dieser Adelshäuser über eine Höhen- oder Wasserburg gebot. Der Begriff Burgenadel wird hier nur zur Abgrenzung gegen diejenigen Familien verwendet, die dem städtischen Patriziat entstammten.

Um die Sprache auf die Egen/Ega zu bringen, so liegt deren Ursprung in der Bürgerschaft von Schwäbisch Hall. Diese bildete eine aus Ministerialen der Staufer oder des Reiches bestehende Eidgenossenschaft, ${ }^{38}$ die freilich keineswegs mit der Gesamtbewohnerschaft gleichzusetzen ist. Genannt seien hier nur die Schultheiß, die sich später von Rinderbach nannten, ${ }^{39}$ und die ebenfalls von dort stammenden Sulmeister, die dann unter dem Namen Senf(f)t erscheinen. Hier lässt sich der interessante Transformationsprozess vom reichsstädtischen Patriziat zur Reichsritterschaft beobachten. Längst hatten sie außerhalb der Reichsstadt Grundbesitz erworben, doch scheint ihnen latent das Gefühl einer gewissen Inferiorität gegenüber dem Burgenadel innegewohnt zu haben. ${ }^{40}$ Als sich der fränkische Adel zur Reichsritterschaft zusammenfand, beeilten sie sich mit dem Anschluss an Ort/Kanton Odenwald als gleichberechtigte und gleichgeachtete Mitglieder. ${ }^{41}$

35 Anders, doch wenig wahrscheinlich Leutwein (wie Anm. 9), S. 161, die Dienheim hätten an die Wollmershausen zu Amlishagen veräußert.

36 Leutweins Angabe, Ebd., S. 161 dürfte wohl zutreffen, wonach Johann Philipp der Jüngere und Heinrich Albrecht von Dienheim zuletzt in Merchingen gewohnt hätten. Dafür spricht, dass Eva Margaretha Katharina die Tochter des Johann Philipp des Jüngeren von Dienheim und der Maria Katharina von Waldhof(en) war; vgl. Johann Gottfried Biedermann: Geschlechts-Register [...] Orts Ottenwald, Kulmbach 1751 (Neudruck Neustadt/Aisch 2000), Tab. CCVI. Die Waldhof(en) gehörten zu den Merchinger Ganerben.

37 Ebd., S. 161.

38 Andreas Maisch: Von der Reichsministerialität zum Stadtpatriziat? Zur Entwicklung des Haller Stadtadels im Mittelalter. In: Hans Gräser (Hg.): Niederadel um Crailsheim. Crailsheim 2012, S. 25-46, hier S. 28.

39 Gerd Wunder: Die Familie von Rinderbach - ein Haller Adelsgeschlecht. In: Der Haalquell. Blätter für Heimatkunde des Haller Landes (1958/1), S. 1-3.

40 Ders.: Von Ega und Egen - ein Beitrag zum Thema Landadel und Stadtadel. In: Archiv für Sippenforschung 49 (1983), S. 41-50, hier S. 47.

41 Vgl. die Mitgliedsliste von Ort Odenwald des Jahres 1566; StAL B 583 Bü 192; zum Verhältnis 
Als Ahnherr der Egen erscheint im Jahre 1273 ein Walter Egeno, der in diesem Jahre eine jährliche Abgabe von 40 Scheffeln Korn vom Kloster Komburg kaufte, die von dessen Gütern in Erlach aufzubringen waren. ${ }^{42}$ In der Folgezeit griffen die Egen über Schwäbisch Hall hinaus. ${ }^{43}$ Ein Hans Egen (gest. 1498) ist erstmals 1453 in Dinkelsbühl bezeugt, wo er mehrfach als Bürgermeister amtierte. 1538 ist ein Egen in Tettnang nachzuweisen. Der einzige seiner Söhne, Daniel, führte die Familie fort. Dessen Sohn Hans amtierte 1530/39 als gräflich-montfortischer Hofmeister und Vogt in Tettnang. Seiner Ehe mit Katharina Arnsperger entstammte die Tochter, die mit Christoph Kröll aus dem Ravensburger Stadtadel verheiratet war, und jener Hans Christoph, der 1562/71 in Tettnang bezeugt ist.

Bis hierher weisen die Entwicklungen der Egen und diejenige der Senf(f)t und Rinderbach kaum Unterschiede auf. Das änderte sich mit einer Eheschließung, deren Tragweite freilich damals noch nicht abzusehen war. Hans Christoph heiratete im Jahre 1554 die Tochter des Marquard V. von Hohenems (gest. 1523), Vogt von Bludenz. ${ }^{44}$ Sie, Amalia, war in erster Ehe mit Six von Schienen zu Gammerschwang verehelicht gewesen. Wie die Verbindung des Hans Christoph mit der Vorarlberger Familie zustande kam, ist unbekannt.

Wurde oben gesagt, die Eheverbindung mit dem Haus Hohenems sei damals in der Bedeutung noch nicht abzuschätzen gewesen, änderte sich das schlagartig mit der Erhebung derer von Hohenems in den Grafenstand. ${ }^{45}$ Die Thronbesteigung des Gian Angelo de Medici am 25. Dezember 1559 als Papst Paul IV. eröffnete dem Haus Hohenems ganz neue Möglichkeiten. Der neue Papst brach mit der antihabsburgischen Politik seines Vorgängers und erkannte die Kaiserwürde des Habsburgers Ferdinand I. an. Dessen Gegenleistung bestand u. a. in der Erhebung der Söhne Wolf Dietrichs und der nunmehrigen Papstschwester Klara von Medici, nämlich Jakob Hannibal, Marx Sittich III. und Gabriel sowie ihres Vetters Marx Sittich II., in den Grafenstand.

Die am 27. April 1560 ausgefertigte Urkunde schloss jedoch nicht den Gatten der Amalia ein. Dieser Hans Christoph von Egen kompensierte seinen Hang zu erhöhtem Sozialprestige, indem er sich fortan von Ega nannte. ${ }^{46}$

von Niederadel und städtischem Patriziat Cord Ulrichs: Vom Lehnhof zur Reichsritterschaft (Vierteljahresschrift für Sozial-und Wirtschaftsgeschichte 134). Stuttgart 1997, S. 61-66.

42 Gerd Wunder: Die Bürgerschaft der Reichsstadt Hall von 1395 bis 1600 (Württembergische Geschichtsquellen 25). Stuttgart, Köln 1956, S. 30.

43 Wunder: Von Ega und Egen (wie Anm. 40).

44 Zur Genealogie vgl. die Stammtafel bei Ludwig Welti: Geschichte der Reichsgrafschaft Hohenems und des Reichshofes Lustenau. Innsbruck 1930.

45 Ebd., S. 90 f.

46 Ebd., S. 47. 


\section{Wolf Heinrich von Ega in Vorarlberg}

Der zweiten Ehe der Amalia von Hohenems entspross unser Wolf Heinrich. Um es deutlich zu sagen - von Kindheit und Jugend, überhaupt für die Jahre vor 1603 in Vorarlberg wissen wir nichts. Kennt man keine urkundliche Nennung, besitzt man dafür eine, wenn auch knappe, narrative Quelle. Der Schüpfer Pfarrer und Historiograph Jakob Ernst Leutwein wusste in seiner 1761 beendeten ,Schüpfer Kirchenhistorie" noch einiges in Erfahrung zu bringen, doch ohne Referenzen zu nennen. ${ }^{47}$ Offenbar besaß er Einsicht in längst nicht mehr erhaltenes Schriftgut der seinerzeitigen Beamten der Ortsherren. Zunächst bezweifelte er, ob es die Familie Ega zu seiner Zeit noch gebe, womit er Recht hatte. Ihre Besitzungen vermutete er am Bodensee, was mit Tettnang wenigstens ungefähr stimmte. Die Zugehörigkeit der Familie zur schwäbischen Reichsritterschaft entnahm er dem Siebmacherschen Wappenbuch. Ferner wusste er von einer Gräfin von Hohenems als der Mutter Wolf Heinrichs zu berichten, woraus er auf hohes Ansehen der Ega in diesem Raum schloss. Wolf Heinrich von Ega - so Leutwein weiter - habe schöne ererbte Gelder den Grafen von Zeil geliehen und sei Teilhaber an den Salzpfannen im Inntal gewesen, deren Nutzung man ihm jedoch schwer gemacht habe. Dies und sein Wegzug seien aufgrund seines evangelischen Bekenntnisses erfolgt. Außerdem hätte er sich - und das trifft zu - verschuldet, als deren Ursachen Leutwein kryptisch „,verschiedene Umstände“ nannte. ${ }^{48}$ Es ist darauf hinzuweisen, dass die Darlehensgewährung sowie Teilhaberschaft an den Salzpfannen ausschließlich durch Leutwein überliefert wird.

Es war eine Zeit sowohl des politischen als auch des wirtschaftlichen Aufstiegs des Hauses Hohenems. ${ }^{49}$ Allein schon die Heiratsverbindungen sprechen für sich, was allerdings nur für die Nachkommen des Wolf Dietrich II. (gest. 10. März 1538) und seiner Gattin Klara von Medici (gest. nach 1577) gilt. Von ihren Söhnen heiratete Jakob Hannibal I. (gest. 1587) Hortensia Borromeo und trat damit in den Verwandtschaftskreis des Mailänder Erzbischofs Carlo Borromeo ein. Marx Sittich III. (gest. 15. Februar 1595), ,völlig ungeistliche(r) Papstnepote und bisherige(r) Condottiere" ${ }^{50}$ erlangte die Würde eines Kardinalbischofs von Konstanz (1561-1589). Von den Söhnen des Jakob Hannibal erreichte Marx Sittich IV. (gest. 9. Oktober 1619) die Würde des Erzbischofs von Salzburg, sodass nach dem Tod des Wolf Dietrich II. (gest. 1604) allein Kaspar (gest. 10. September 1640) diesen Zweig fortführte.

Die Verbindung zu den Medici und Borromeo verweist auf streng katholische Ausrichtung. Das Haus Hohenems gehörte der Gruppe der katholischen-schwä-

47 Leutwein (wie Anm. 9), S. $164 \mathrm{f}$.

48 Ebd., S. 164.

49 Welti: Geschichte (wie Anm. 44), S. 90 u. 99 f.; auch Karl Heinz Burmeister: Geschichte Vorarlbergs. Ein Überblick. Wien 1989, S. 116-122.

50 Eike Wolgast: Hochstift und Reformation. Studien zur Geschichte der Reichskirche zwischen 1517 und 1648. Stuttgart 1995, S. 163. 
bischen Grafen an, die man zu Recht so gekennzeichnet hat, sie bildeten ,militärisch und politisch eine Art Reserve der katholischen Partei im Reich im Gefolge der führenden Dynastien der Habsburger und der bayerischen Wittelsbacher". 51

Damit einher ging die wirtschaftliche Konsolidierung durch eine kluge Familienpolitik. ${ }^{52}$ War das Haus Hohenems vor allem durch Besitzteilungen belastet, beugte Graf Jakob Hannibal I. zunächst weiterer Zersplitterung des Familienbesitzes durch Schaffung eines Majorats für seinen ältesten Sohn Kaspar vor. Dieser hätte auch der Erbe des Gesamthauses sein können, wenn nicht...

Damit ist man beim zweiten Zweig derer von Hohenems, der an dem kometenhaften Aufstieg nicht teil hatte. Da waren die Kinder des 1533 verstorbenen Marquard V. und seiner Gattin Veronika von Neideck: Mark Sittich II., Vogt zu Bludenz (gest. 1565), in erster Ehe verheiratet mit Eva von Dankertschweil, in zweiter mit Eva von Thun. Einer dieser Ehen entstammte der 1603 verstorbene Hans Christoph, verheiratet mit Maria von Paumgarten zu Hohenschwangau (gest. 1633), mit dem dieser Zweig erlosch. Die Schwester, Amalia, heiratete nach dem Tod des Sixt von Schienen zu Gammerschwang den Hans Christoph von Ega. Dieser Ehe entstammte Wolf Heinrich.

Auch wenn wir nicht den geringsten unmittelbaren Quellenbeleg besitzen, ist das Urteil legitim, er habe innerhalb des Hohenemsischen Familienverbandes wie ein Fremdkörper gewirkt. Zwei Gründe lassen sich dafür namhaft machen. Die in den Grafenrang aufgestiegenen und mit bedeutenden Persönlichkeiten verschwägerten Hohenemser sahen in Ega einen unter ihrem Rang stehenden Ritteradligen. In den noch vorzustellenden Verkaufsdokumenten tritt diese ständische Distinktion deutlich genug hervor: Hier der Hoch- und Wolngeborne Graf Caspar zu der Hohenembs und Gallara, dort der Edel und Vest Wolf Heinrich von Ega, der also nicht in die Erhebung in den Grafenstand miteinbezogen worden war. Viel später hatte Wolf Heinrich in seiner neuen Heimat Schüpf in nächster Nähe Gelegenheit, eine ständische Mesalliance zu beobachten.

Nicht leicht zu beantworten ist das konfessionelle Problem. Leider gibt es für die Zeit vor der Einheirat in die Ganerbschaft Schüpf nicht den geringsten Hinweis auf Egas religiöse Haltung. Geht man von dem aus, was wir über seine Schüpfer Zeit wissen, ist er evangelisch gewesen. Es gibt durchaus Beispiele für die Akzeptanz konfessioneller Abweichung von Angehörigen ein und derselben Familie, für die Hohenems ist dies angesichts ihrer Verwandtschaft, des sozialen Aufstiegs und ihres politischen Ehrgeizes allerdings mehr als unwahrscheinlich. Andererseits ist mit letzter Sicherheit nicht auszuschließen, dass - und auch da-

51 Anton Schindling: Das Ende des Konzils von Trient 1563, das Heilige Römische Reich, die Habsburger und die Reichskirche. In: Würzburger Diözesangeschichtsblätter 77 (2014), S. 27-58, hier S. 56.

52 Welti: Geschichte (wie Anm. 44), S. 99. 
für gibt es Beispiele - der Glaubenswechsel Bedingung für die Schüpfer Heirat gewesen ist. Wenn ersterer Erklärung hier der Vorzug gegeben wird, bleibt dennoch eine gewisse Unsicherheit.

\section{Erbschaft und Verkauf}

Zwischen den beiden Zweigen derer von Hohenems muss es Spannungen von solcher Intensität gegeben haben, die Graf Hans Christoph bewogen, den anderen, d. h. Kaspar in seinem Testament zwar nicht zu übergehen, so doch nicht als Haupterben einzusetzen. Als er am 17. Februar 1603 ohne legitime Erben verstarb, vermachte er seinen Besitz Wolf Heinrich. ${ }^{53}$ Ob er, der damals ja schon viele Jahre im Schüpfergrund wohnhaft war, von diesem Ereignis überrascht wurde oder erst seit der Testamentserstellung davon wusste, ist nicht bekannt. Überhaupt bleiben, was die Verkaufsvorgänge betrifft, viele Fragen unbeantwortet.

Es konnte Wolf Heinrich wohl keinen Augenblick verborgen bleiben, dass er das Erbe nicht würde halten können. Er trat deshalb die Reise nach Vorarlberg an, wo er am Dienstag (Zinstag), dem 4. November, zunächst Kontakt mit dem Landvogt der Vogtei Reineck, Adrian Ziegler von Zürich, aufnahm. ${ }^{54}$ Dessen Bericht an Graf Kaspar vom 6. November 1603 lässt noch etwas von dem Schock erahnen, den das Testament ausgelöst hatte, wenn er nämlich von Wolf Heinrich als dem angemaßten Erb spricht. Aus seinem Schreiben geht nicht eindeutig hervor, ob dieser das Erbe nur Graf Kaspar angeboten hatte, sondern auch anderen Interessenten. Wie dem auch sei, bislang war man sich über den Preis nicht einig geworden. Dennoch wolle er es, um Streit, Weitläufigkeit sowie discontirte Kosten zu vermeiden, weiterhin dem Grafen anbieten, wie der Landvogt hinzufügte. Wahrscheinlich ahnte zu diesem Zeitpunkt noch keiner der drei, wie es damit bestellt war.

Bereits am 2. Dezember 1603 trafen sich Ega und Graf Kaspar zur abschließenden Verhandlung. Hinzugezogen hatten sie (oder nur Graf Kaspar?) die Geheimen kaiserlichen Räte Johann Ludwig von Ulm zu Wangen und Marbach und den Vogt von Bregenz und Hoheneck Johann Werner von Raitenau zu Hofen und Lochau. Man einigte sich wie folgt:

1. Ega übergibt dem Grafen Kaspar alle von seinem Vetter ererbten Güter mit Einkünften und Rechten. Diese werden in der eigentlichen Verkaufsurkunde aufgeführt: ${ }^{55}$ Dessen Ansprüche wegen der Besserungen an dem Vorhof zu Ems;

53 Ludwig Welti: Graf Kaspar von Hohenems 1573-1640. Innsbruck 1963, S. 27; Ders.: Geschichte (wie Anm. 44), S. 99.

54 Vorarlberger Landesarchiv. Hohenemser Archiv 033,07 II 0511. Egas Güterverkauf an Graf Kaspar um 37.000 fl und Hohenems Reichsgrafschaft 8709. - An dieser Stelle hat Verf. Herrn Universitätsdozent Dr. Manfred Tschaikner, Vorarlberger Landesarchiv, zu danken.

55 Vorarlberger Landesarchiv. Hohenems Reichsgrafschaft 8709. 
alle Ansprüche an Güter, Gefälle und Leute in der Grafschaft Ems; dessen Hälfte am Weingarten an der Klaus; dessen Hälfte an der Alpe Süns in der Herrschaft Feldkirch und alle Rechte und Gefälle in eben dieser Herrschaft; dessen Hälfte an der Alpe Zürs in der Herrschaft Sonnenberg; dessen Hälfte am Reichshof Lustenau, Widnau und Haslach mit allen Gütern und Rechten; dessen Hälfte an den Weingärten im Rheintal zu Monstein, Tobel und Hausen; alle Steuern an den Höfen Altstätten, Marbach und Bernang sowie dessen Hälfte an dem Haus zu Lindau. Ausgenommen sind die $13.000 \mathrm{fl}$, die in der Tirolischen Kammer liegen sowie die von Ega beanspruchte Mailändische Pension; ferner Farnis, Mobilien und drei Harnische.

2. Der Kaufpreis beträgt $37.000 \mathrm{fl}$ rheinisch. Davon abgezogen werden $10.000 \mathrm{fl}$, die Graf Kaspar von diesem Erbe zugefallen sind und die bei ihm verbleiben. Die übrigen $27.000 \mathrm{fl}$ sollen an einem sicheren Ort hinterlegt werden, sodass keine Partei ohne die andere Zugang hat. Davon sollen alle Schulden des Hans Christoph beglichen werden. Was übrig bleibt, wird Ega zugestellt werden. Wenn er aber zwischenzeitlich Geld benötigt, sollen ihm zu Innsbruck $300 \mathrm{fl}$ gereicht werden.

3. Ega ist befugt, die heurigen Gefälle von allen Gütern einzuziehen, ausgenommen diejenigen zu Ems.

4. Ega wird Rosina Embserin und Amalia Loring in Erfüllung des Testaments der Frau von Thun $3000 \mathrm{fl}$, die auf der Herrschaft Bludenz liegen, versichern und übergeben, doch so, dass, wenn das Rosinle zuvor stürbe, das Geld wieder an ihn zurückfällt.

Über diesen letzteren Punkt gewinnt man etwas mehr Klarheit, durch einen Legbrief vom 1. Januar 1604. Darin erklärte Graf Kaspar, dass Ega einen Hauptbrief, die auf der Herrschaft Bludenz liegenden $6000 \mathrm{fl}$ betreffend, in Händen hat. Eine Hälfte dieser Summe hat Ega dem Cyprian von Thun zu entrichten versprochen, die andere den beiden Mädchen. Es gab aber noch Ansprüche anderer. Mit den von Thun in Innsbruck - die zweite Gattin von Hans Christophs Vater war Eva von Thun - hat er sich ebenso geeinigt wie mit Maria von Paumgarten, der Witwe des Hans Christoph. Als Entschädigung für den Zehnten zu Lustenau sprach er ihr einen Diamanten, Silbergeschirr, eine Kutsche und vier Pferde zu. ${ }^{56}$ Um eine letzte Güterverschiebung anzuführen, so veräußerte Wolf Heinrich zwei Tage nach dem Hauptverkauf aus dem Erbe einen das Reithen genannten Besitz zu Hohenems um $320 \mathrm{fl}$ dem Grafen Kaspar. ${ }^{57}$

Um eine abschließende Bewertung der Vorgänge vorzunehmen - soweit das bei der Quellenlage überhaupt möglich ist - sind noch drei weitere Vorgänge anzuführen. Ega bekannte am 5. Dezember 1603 - er weilte damals noch in Vorarl-

56 Welti: Graf Kaspar (wie Anm. 53), S. 28.

57 Vorarlberger Landesarchiv. Hohenemser Archiv 033,07 II 0511. 
berg - einem Feldkircher Bürger $870 \mathrm{fl} \mathrm{zu}$ schulden. Diese Summe wird mit Zustimmung des Grafen Kaspar von den 37.000 fl abgezogen. ${ }^{58}$

Ließ sich dies offenbar problemlos lösen, hatte Ega sich mit zwei Ansprüchen anderer auseinanderzusetzen, deren Ausgang man nicht kennt. Im Dezember des Jahres 1604 stellten Geschwister und Schwäger des verstorbenen Bludenzer Bürgers Rudolf Embser als arme kinderreiche Hausleute an Ega als Universalerben die Forderung nach Erstattung von neun Jahren Besoldung (150 fl) als Kämmerling des verstorbenen Hans Christoph. ${ }^{59}$ Diese rückwirkende Zahlung sollte durch ein von Felicitas von Thun bei der Vogtei Bludenz angelegtes Kapital erfolgen, dessen Zinsen Ega bezogen hätte. Ob diese Forderung von Erfolg gekrönt war, ist fraglich.

Den Ausgang der dritten dieser Forderungen, die Ega über Jahre hinweg verfolgte, kennen wir ebenfalls nicht. Jedenfalls wandte Ega sich am 21. Juni 1606 von Oberschüpf aus an Graf Kaspar um Unterstützung. Johann Rem, der ehemalige Hofmeister des verstorbenen Hans Christoph, forderte von ihm, Ega, für $30 \mathrm{Jah}$ re ausstehende Besoldung, und zwar $100 \mathrm{fl}$ pro Jahr. Rem erhielt aber - so Ega - bis 1594 von dem verstorbenen Grafen seinen jährlichen Lohn. Dann - und hier wird das Ganze sehr unklar - übergab man ihm die mit 3000 fl veranschlagte Mühle zu Lustenau, weshalb Rem also für 21 Jahre Besoldung forderte, die ihm nicht zustand. Dies sei Graf Kaspar bekannt, denn jüngsthin habe Ega den Grafen dahingehend unterrichtet. Da die Mühle aber dessen Jurisdiktion unterstehe, möge dieser dafür Sorge tragen, ihm die Mühle wieder zu übertragen oder eine Einigung herbeizuführen, um nicht gegen Rem klagen zu müssen. Es sollte nicht die letzte Reise nach Vorarlberg gewesen sein.

Stellt man das Erbe, das einen Verkaufswert von 37.000 fl besaß, den Darlehensaufnahmen und Rückzahlungsschwierigkeiten im Schüpfergrund gegenüber, sieht man sich auf den ersten Blick einem eklatanten Widerspruch gegenüber. Bei allen Abzügen und Abgeltung von Ansprüchen anderer müsste doch eine stattliche Summe in Händen Wolf Heinrichs verblieben sein. Wie vereinbart sich das mit den Finanzproblemen, wie sie in Schüpf offenbar werden? Anscheinend wurde der Verkaufserlös weitgehend von der Schuldentilgung des Erblassers aufgefressen. Es ist sicher nicht ganz falsch, hier von einem Danaergeschenk zu sprechen. Ob es bei all diesen Transaktionen einen wirklichen Nutznießer gab, ist nur schwer zu entscheiden, doch wenn, kann es nur Graf Kaspar gewesen sein. Zudem gewinnt man den Eindruck - das aber mit aller Vorsicht: Wolf Heinrich sei ihm nicht gewachsen gewesen.

58 Ebd.

59 Welti: Graf Kaspar (wie Anm. 53), S. 29 A 1. 


\section{Wolf Heinrich von Ega im Schüpfergrund: Status und Familie}

Mit obiger Darstellung ist man den Ereignissen zeitlich vorausgeeilt, denn die Dokumente um die Vorarlberger Vorgänge unterzeichnete Wolf Heinrich von Ega stets als zu Ober- und Unterschüpf gesessen. In den Schüpfergrund war er 1594 durch die Heirat mit Ruf(f)ina geborener von Leyen, der Witwe des am 13. Dezember 1589 verstorbenen Ägidius Reinhard von Dienheim, gelangt. ${ }^{60}$ Es spricht einiges für Leutweins Angabe, dass die Hohenemser ihn nicht zuletzt aus konfessionellen Gründen aus Vorarlberg hinausgedrängt haben. Doch wie kam die Eheverbindung in den Schüpfergrund zustande und das in eine wirtschaftlich nicht gerade auf Rosen gebettete Adelsfamilie? Bekannt ist, dass Eheverbindungen beim Adel in vielen Fällen durch Mediatoren angebahnt wurden, die man entweder , mit der Suche ${ }^{6}$ beauftragte oder die auf eigene Rechnung handelten. ${ }^{61}$ Auch auf diese Frage gewähren die Quellen keine Antwort.

Mit der Heirat erlangte er aber die Mitgliedschaft im Ort Odenwald der fränkischen Reichsritterschaft. Daraus wird ersichtlich, dass Einheirat in eine dem Ort inkorporierte Familie die Aufnahmekriterien erfüllte. Das berührt die Frage, ob ihn das auch zum Ganerben machte. Antwort auf diese Frage erlauben die hohenlohischen Aufbietungen an den Lehnadel.

Erstmals im Jahre 1583 forderte Graf Wolfgang von Hohenlohe seine Lehnsleute auf, sich wegen ereignender Kriegsleufften bewaffnet bereitzuhalten. ${ }^{62}$ Es ist bemerkenswert, wie sehr die Krise am Niederrhein ${ }^{63}$ so weit entfernte Gebiete beunruhigte. Die folgenden Lehnaufgebote zeichnen geradezu die Stufen der sich steigernden krisenhaften Zuspitzung im Reich nach. Das gräfliche Schreiben vom 28. Mai $1608^{64}$ war die Reaktion auf die bayerische Annexion der Reichsstadt Donauwörth. ${ }^{65}$ Das folgende Landrettungsaufgebot nannte u.a. Eberhard, Albrecht und Philipp Adam von Dienheim. ${ }^{66}$ Am 22. März 1610 erklärte Albrecht (III.) von Dienheim der Ältere zu Unterschüpf auch im Namen seines Bruders Eberhard mit tauglichem Pferd, Rüstung und Knecht der gräfli-

60 Leutwein (wie Anm. 9), S.164.

61 Annette Baumann: Eheanbahnung und Partnerwahl. In: Siegrid Westphal u.a.: Venus und Vulcanus. Ehen und ihre Konflikte in der Frühen Neuzeit. München 2011, S. 25-87.

62 Christian Ernst Hanßelmann: Diplomatischer Beweiß, daß dem Hause Hohenlohe die Landeshoheit $[\ldots]$ nicht etwan in dem sogen. großen Interregno oder nach solchen Zeiten erst, zu theil worden, sondern demselben schon lang vorher zugestanden. Nürnberg 1751, S. 545.

63 Volker Press: Kriege und Krisen. Deutschland 1600-1715. München 1991, S. 174 f.; Franz Bosbach: Köln. Erzstift und Freie Reichsstadt. In: Anton Schindling / Walter Ziegler (Hg.): Die Territorien des Reichs im Zeitalter der Reformation und Konfessionalisierung. Bd. 3: Der Nordwesten. Münster ${ }^{2} 1995$, S. 58-84, hier S. 74 f.; Axel Gotthard: Konfession und Staatsräson. Die Außenpolitik Württembergs unter Herzog Johann Friedrich. Stuttgart 1992, S. 61-83, 94-120.

64 Hanßelmann: (wie Anm. 62), S. 552, Nr. CCXXXVII.

65 Moriz Ritter: Deutsche Geschichte im Zeitalter der Gegenreformation und des Dreißigjährigen Krieges (1555-1648), Bd. 2. Stuttgart 1895, S. 213-215.

66 Hanßelmann (wie Anm. 62), S. 558f, Nr. CCXLVII. 
chen Aufforderung nachkommen zu wollen. ${ }^{67}$ Diesesmal war es der sich zum europäischen Konflikt zusammenfallende Jülische Erbfall, der den Aufruf veranlasste. ${ }^{68}$ Nach Ausbruch des Dreißigjährigen Krieges setzte Graf Georg Friedrich die Landrettungsbemühungen energisch fort. ${ }^{69}$ Von Angeltürn aus erklärte Johann Philipp von Dienheim auch für seinen Bruder am 9./19. Juni 1619 seine Bereitschaft. ${ }^{70}$ Im Verzeichnis eben dieses Jahres der von Graf Georg Friedrich aufgebotenen Lehnsleute finden sich die Namen Albrecht Nikolaus, Hans Reinhard, Hans Philipp und Albrecht von Dienheim. ${ }^{71}$

So sehr diese Maßnahmen angesichts der Kriegsgefahren auch antiquiert gewesen sein mögen, sie sind für unseren Zusammenhang aufschlussreich. Genannt werden Angehörige der Dienheim, doch nur einmal, nämlich 1619, Wolf Heinrich von $\mathrm{Ega}^{72}$ und das im Landrettungsaufgebot. Zieht man Belehnungen heran, so empfingen Egas Gattin und deren Brüder Hans Reinhard und der Johanniterkomtur Albrecht Nikolaus von Dienheim im Jahre 1613 die hohenlohischen Lehen - zwei Teile am Zehnten zu Neidlingen und Dachhof bei Assumstadt in der Harenbach, den Halbteil am Weinzehnten zu Oberschüpf, ein Hof zu Klepsau, zwei Höfe in Ailringen, Gülten in Unterschüpf sowie die Kirchenpatronate in Unterschüpf und Schweigern -, wobei Wolf Heinrich als Träger fungierte. ${ }^{73}$ Da er selbst nicht als Lehnsmann genannt wurde, kann das nur heißen, dass er keine hohenlohischen Lehen trug, er also nicht Ganerbe gewesen ist.

Dem steht aber eine andere Aussage entgegen. Im Zuge der vom Nürnberger Exekutionsausschuss zum Abschluss zu bringenden negotia remissa oder ,zurückgestellte Materien“, d.h. der im Osnabrücker Friedensinstrument unerledigten Streitfragen, ${ }^{74}$ gehört der Zwist zwischen Johann Kaspar von Stetten zu Kocherstetten und den Grafen von Hatzfeldt ${ }^{75}$ um den Kirchenpatronat von Schüpf und Uiffingen. Im Schreiben des Stetten an den Ritterhauptmann Johann Kaspar von Herda vom 9. August 1649 heißt es, es gehe um den Streit zwischen ihm und den Ega als Mitganerben. In einem Memorial der Condominorum des Schüpfer

67 Ebd., S. 561, Nr. CCLII.

68 Press (wie Anm. 63), S. 174-184.

69 Zur Politik des Grafen vgl. Frank Kleinehagenbrock: Die Grafschaft Hohenlohe im Dreißigjährigen Krieg (Veröffentlichungen der Kommission für geschichtliche Landeskunde in Baden-Württemberg. Reihe B, 153). Stuttgart 2003, S. 38 f.

70 Hanßelmann (wie Anm. 62) S. 566, Nr. CCLXI.

71 Ebd., S. 568 f. Nr. CCLXIV; die Namen auch bei Leutwein (wie Anm. 9), S. 161.

72 In der Liste von 1619 wird Ega (Eger) erwähnt, doch enthält sie auch Namen, die zuvor nicht verzeichnet gewesen sind. Offensichtlich versuchte der Graf das Aufgebot nach Möglichkeit zu erweitern.

73 HZA GA 20 Gemeinschaftliches Lehenarchiv Schublade XXIV Nr. 5, Dienheim. Diese Stücke empfing im Jahre 1659 Johann Philipp von Dienheim; GA 20 Schublade XXIV Nr. 8, Dienheim.

74 Antje Oschmann: Der Nürnberger Exekutionstag 1649-1650 (Schriftenreihe der Vereinigung zur Erforschung der Neueren Geschichte 17). Münster 1991.

75 Oliver Fieg (Bearb.): Archiv der Freiherren von Berlichingen zu Jagsthausen (Inventare der nichtstaatlichen Archive in Baden-Württemberg 25/1). Stuttgart 2012, S. 701, Nr. 3717. Für die Einsichtnahme in die Archivalie hat Verf. Freiherrn Konrad von Berlichingen zu danken. 
Grundts werden als Patronatsherren der Uiffinger Kirche vor unvordencklichen Jahren die Herren von Rosenberg und Dienheim und jetzt Ega und Stetten genannt.

Mit dem Tod des Sohnes von Wolf Heinrich im Jahre 1639 aber waren die Ega im Mannesstamm erloschen. Wahrscheinlich bezieht sich die Angabe auf die im Laienstand verbliebene Ega-Tochter Anna Veronika (s. u.). Da es sich bei den Patronaten um Erblehen handelte, war der Übergang an weibliche Nachkommen durchaus möglich. Man wird jedoch einräumen müssen, dass die Rechtsverhältnisse im Schüpfergrund zuvor schon umstritten waren und in den Wirren der Spätphase des Dreißigjährigen Krieges mögen sie vollends unklar geworden sein.

Die letztlich entscheidende Aussage liefert ein Bildwerk: Anlässlich des Centenariums der Reformation wurde in der Unterschüpfer Kirche eine neue Kanzel aus Stein errichtet. Das Corpus trägt die Wappen der Orts- und zugleich Kirchenherren Dienheim, Stetten und Rosenberg - nicht Ega.

Versucht man den Status des Wolf Heinrich von Ega zu definieren, ist er am ehesten - sit venia verbo - als derjenige eines ,Prinzgemahls` zu beschreiben. Er hatte keinen Anteil an der Herrschaft, hatte jedoch die rechtliche Vertretung der Gattin und dann der Kinder inne.

Nach diesem Exkurs zurück zur familiären Situation. Beide Ehen Ruf(f)inas überschatteten Unglücksfälle. Ein Sohn aus erster Ehe, Wilhelm Albrecht, verunglückte tödlich durch einen Sturz im Treppenturm des Oberschüpfer Schlösschens, ein Söhnchen aus der zweiten, Wolf Georg, stürzte von einer Mauer in einen kleinen See beim Schlösschen und ertrank. ${ }^{76}$ Aus der ersten Ehe blieb ihr nur Hans Reinhard, zu dem schon einiges gesagt worden ist. Zum einzigen männlichen Nachkommen aus der zweiten Ehe, Philipp Ludwig, wird ebenfallls noch etwas zu sagen sein.

Der Nachweis einer Tochter Wolf Heinrichs gründet auf der Ahnenprobe auf dem Grabdenkmal des Johann Philipp von Hoheneck in St. Burkhard zu Würzburg. ${ }^{77}$ Bis auf eine Verbindung zum Haus Hardheim ist von Verwandtschaftsbeziehungen so gut wie nichts bekannt. Dissense mit Würzburg über die Türkensteuer ließen sich am 26. November 1598 vertraglich beilegen. Als Rechtsbeistände des Georg Wolf von Hardheim fungierten dabei neben einem Juristen der mainzische Forstmeister Emmerich von Hedersdorf, Wolf Christoph Hund von Wenkheim und Wolf Heinrich von Ega. ${ }^{78}$ Als Hochzeitsgeschenk für die dritte Ehe des Georg Wolf von Hardheim stifteten Wolf Heinrich und Ruf(f)ina einen vergoldeten Becher, dessen Deckel ein Mänlin, wohl eine mythologische oder Heiligenfigur, be-

76 Leutwein (wie Anm. 9), S. 165. Bei Johann Maximilian Humbracht: Die Höchste Zier Teutsch-Landes. Und Vortrefflichkeit des Teutschen Adels. Frankfurt 1707,Taf. 18 irrtümlich Johann Wigand, gest. 1590 .

77 Leutwein (wie Anm. 9), S. 164.

78 Jakob Albert Prailes: Die Einführung der Reformation in Hardheim (Amt Buchen). In: Freiburger Diözesanarchiv N.F. 8 (1905), S. 258-341, hier S. 310 f. 


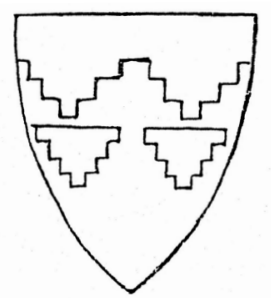

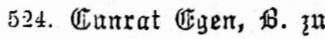
(1)all. 1369.

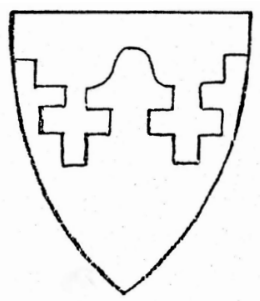

525. S. Egenonis, \$dult-

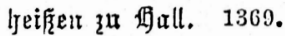

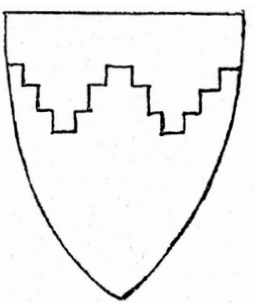

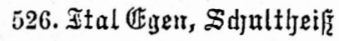
ża fiall. 1379.

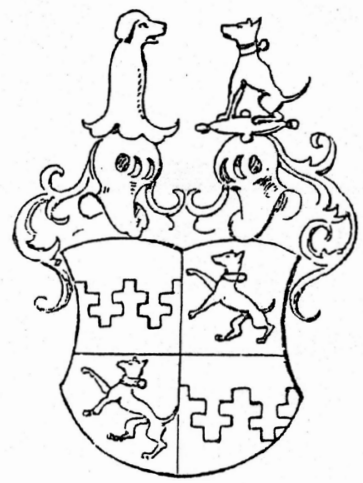

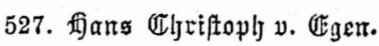
1566.

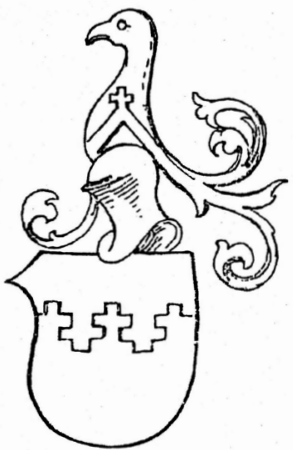

528. willelur 估gen, Bürgex

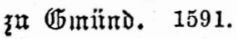

Die verschiedenen Egen-/Ega-Wappen aus Siebmachers Wappenbuch, (Vorlage: Siebmacher, wie Anm. 4).

krönte. ${ }^{79}$ Die Braut des Hardheim, Anna Philippa von Leyen, war eine Schwester der Ruf(f)ina. Eine weitere Verbindung zum Hause Hardheim lässt sich am Namen des ertrunkenen Kindes festmachen. Pate des unglücklichen Wolf Georg müsste aufgrund der Namengebung Georg Wolf von Hardheim gewesen sein.

Es gibt noch einen, wenn auch nicht ganz gesicherten Anhaltspunkt für das familiäre Geflecht. Die St. Gumbertkirche in Ansbach verwahrt das Epitaph des 1617 verstorbenen Georg Eberhard Kroll von Krollenstein. In der Ahnenprobe findet sich das Egasche Wappen. Nach der Anordnung muss es sich um dasjenige einer Großmutter des Verstorbenen handeln, die sich sonst in keiner Quelle findet. Wie Leutwein annahm, handelte es sich um eine Schwester Wolf Heinrichs. ${ }^{80}$ Sollte das wirklich zutreffen, hätte Wolf Heinrich nicht allein Vorarlberg verlassen.

79 StAL B 94a Familienarchiv von Hartheim, Bü 2: Inventarium / Weylandt des Gestrengen Edlen / unnd Vesten Georg Wolffen von / und zu Hartheim und Dommeneckh / seeligen verlassenschafft, fol. 52 r.

80 Leutwein (wie Anm. 9), S. 164. 


\section{Wolf Heinrich von Ega im Schüpfergrund: ökonomische Situation}

In drei allerdings unterschiedlich dokumentierten Bereichen, nämlich als Bauherr, bei Besetzung der Kirchenstellen sowie in finanziellen Angelegenheiten gewinnt der Name Ega so etwas wie Konturen.

Bautätigkeit gehörte zum Lebensgefühl des Edelmanns, und Ega macht hier keine Ausnahme, wenn ihm auch enge Grenzen gesetzt waren. Leutwein überliefert die von ihm 1616 in Auftrag gegebene Errichtung eines Seitengebäudes zum Oberschüpfer Schlösschen als Wohnung für seinen Beamten und Hofbauern. ${ }^{81}$ Hier erfährt man, dass er (namens der Gattin) einen Hof im Eigenbau betrieb, während seine anderen grundherrlichen Einnahmen aus den Natural- und Geldabgaben der Bauern herrührten, die das Land in Erbzinsleihe innehatten. Offenbar war dem Beamten auch der Einzug der Gefälle anvertraut und er übte so etwas wie eine Kontrollfunktion aus.

An dieser Stelle sei etwas vorausgeschickt, das im Zusammenhang des Patronatsrechts noch beschäftigen wird und das mit der wappengezierten Kanzel schon angeklungen ist. Seit Jahren sahen die Ganerben dem Reformationscentenarium entgegen. Über dessen Ablauf vermochte Leutwein nichts in Erfahrung zu bringen, doch an der an einer Emporensäule angebrachten Jahreszahl 1617 vermochte er den Umbau der Unterschüpfer Kirche als architektonischen Beitrag zu diesem Jubiläum abzulesen. ${ }^{82}$ Sie erfuhr einen Anbau in Richtung des Dorfes, wodurch sie den charakteristischen Winkelgrundriss erhielt. ${ }^{83}$ Der Corpus der neuerrichteten Kanzel trägt - es ist schon gesagt worden - die Wappen der drei Ganerben Rosenberg, Stetten und Dienheim, wobei Letzteren die ehrenvolle mittlere Position zugestanden wurde. Diese Anordnung markierte den Abschluss gewisser Dissense (s. u.) und trug der Tatsache Rechnung, dass den Dienheim das Jus patronatus zukam.

Die Freude an Status und evangelischer Repräsentation dürften allerdings unter dem Schatten des Finanziellen gestanden haben. Die Aufteilung der Baukosten, ob gedrittelt oder anders aufgeteilt, oder ob Spendengelder zum Bau beitrugen, ist unbekannt. Man wird aber doch soviel behaupten dürfen, dass den beiden Rosenberg die Finanzierung leicht, den Stetten nicht schwer fiel, doch für Dienheim bzw. Ega mit erheblichen Problemen verbunden war.

Bekanntlich differierten die Vermögensverhältnisse bei der Ritterschaft beträchtlich. ${ }^{84}$ Für diejenige des Orts Odenwald reicht sie von den Rosenberg der letzten

81 Ebd., S. 165.

82 Jakob Ernst Leutwein: Schüpfer Kirchenhistorie. Zweytes Buch, Sectio I., S. 42 f.; Oechelhäuser (wie Anm. 31), S. 214 nennt dieses Jahr als an einer Holzdecke befindlich.

83 Heinrich Niester: Die evangelische Kirche in Unterschüpf. In: Nachrichtenblatt der Denkmalpflege in Baden-Württemberg, 4 (1961), S. 68-74.

84 Dazu Gert Kollmer: Die schwäbische Reichsritterschaft zwischen Westfälischem Frieden und Reichsdeputationshauptschluss. Untersuchung zur wirtschaftlichen und sozialen Lage der Reichsritterschaft in den Ritterkantonen Neckar-Schwarzwald und Kocher (Schriften zur südwestdeutschen Landeskunde 131). Stuttgart 1979. 
Generation als Spitzenreiter, die imstande waren, selbst Fürsten mit Darlehen unter die Arme zu greifen, bis zu solchen, die kaum mehr als ein wohlhabender Bauer besaßen. Die Vermögensverhältnisse und deren Vergleich bei der Reichsritterschaft Ort Odenwalds sind nur über deren Steuerleistung in die kantonale Kasse (Truhe) zu fassen. Was die Dienheim angeht, ist man in der nicht ungünstigen Situation, dass die Einnehmer des Orts Odenwald, Hans Gottfried von Berlichingen und Hans Heinrich von Heusenstamm, beim Rittertag 1578 in Mergentheim die Rittersteuer der Angehörigen des Orts verzeichneten. ${ }^{85}$ Andererseits sind die Angaben zu den Dienheim alles andere als klar: Für die Eigenerben werden für den vierten Teil der Schüpfer Güter Albrechts von Rosenberg 75 fl genannt, für die Lehnserben 362 fl. Mit gebotener Vorsicht wird man als Lehnserben, d.h. der Mannlehen, die Rosenbergischen Vettern annehmen, als Eigenerben die Cognaten, also Stetten zu Kocherstetten und Leyen bzw. Dienheim. Die Steuerhöhe der Letzteren ist dennoch nicht genauer zu bestimmen, weil aus der Steuersumme der nicht bekannte Anteil der Stetten herausgerechnet werden muss. Dennoch traten die Leyen bzw. Dienheim ein Erbe an, das sich durchaus sehen lassen konnte. Angesichts der verworrenen Familien- und Besitzverhältnisse und auch wenig ausgeprägter ökonomischer Kompetenz entwickelte sich die finanzielle Situation der Ruf(f)ina und Wolf Heinrichs wenig erfreulich.

Auf Philippi und Jakobi des Jahres 1599 (1. Mai) lieh ihm als Träger seiner Gattin Graf Ludwig von Löwenstein (1530-1611) aus dem Fonds des Chorstifts Wertheim 1000 fl guter gangbarer Müntz Fränckischer Währung, den Gulden zu 15 Batzen oder 60 Kreuzer. Die Summe war auf Philippi und Jakobi des Jahres mit $50 \mathrm{fl}$ zu verzinsen, die Rückzahlung war auf vier Jahre festgesetzt. Abgesichert war das Darlehen durch den Großen und Kleinen Getreide- und Weinzehnten zu Ober- und Unterschüpf; als Bürgen fungierten Christoph Wolf Hund von Wenkheim zum Altenstein, Georg Wolf von Hardheim und Hans Philipp Hund von Wenkheim. Ausbezahlt wurden zunächst nur 800 fl, die Restsumme erst am 29. Dezember $1600 .{ }^{86}$

Die folgenden Jahre bestimmten ständige Bitten um Verlängerung des Darlehens und Stundung der Zinsen das Bild. ${ }^{87}$ Philippi und Jakobi 1604 hatten sich die nicht bezahlten Zinsen auf $240 \mathrm{fl}$ aufgestaut. Endlich am 11. Mai 1605 quittierte das Chorstift 550 fl samt Zinsen, wonach das Spiel weiterging und Ega für das Verbleibende um Verlängerung bat. Dabei nannte er ihm in Vorarlberg zustehende Zinsen in Höhe von 6000 fl, deren Auszahlung - wie er erklärte - man ihm verweigere. Was diese Vorarlberger Gelder betrifft, erlaubt die Quellenlage keine auch nur annähernd gesicherte Aussage. Vergeblich bat er am 17. März 1606 den Deutschmeister Erzherzog Maximilian um Hilfe. ${ }^{88}$ Auf die Mahnung vom

85 StAL B 583 Bü 192 fol. 48r-56v.

86 StAWt GA 45/20: Quittung über die 200 fl.

87 StAWt Rep. 46 Nr. 57.

$88 \mathrm{Zu}$ ihm Heinz Noflatscher: Maximilian der Deutschmeister 1558-1618 (Quellen und Studien zur Geschichte des Deutschen Ordens 11). Marburg 1987. 
24. Juni 1606, wandte er sich an Gabriel Dionis von Schellenberg, den österreichischen Verwalter der Grafschaften Bludenz und Sonnenberg, wegen ihm dort noch zustehender $1500 \mathrm{fl}$. Er habe sich sogar persönlich nach Feldkirch begeben, nur um dort zu erfahren, dass Schellenberg kurz zuvor verstorben war. Die Witwe erklärte ihm, vor der Eröffnung des Testaments durch die Kammer zu Innsbruck nichts unternehmen zu können und er möge sich bis Laurentii oder Bartholomei gedulden. Daraufhin sei er abgereist.

Am 22. März 1607 versicherte er dem Grafen auf erneut ergangene Mahnung, er täte nichts lieber, als auf kommenden 1. Mai die $500 \mathrm{fl} \mathrm{zu}$ erlegen, doch benötige er weiteren Aufschub. Am 15. Mai des Jahres teilte er dem Grafen mit, ein gewisser Konrad Meyer zu Balbach hätte ihm ein Darlehen von $600 \mathrm{fl}$ zugesagt. Offensichtlich hat sich auch das zerschlagen. Da die bisherigen Bürgen verstorben waren, fand sich in der Person des Hans Philipp Hund von Wenkheim ein Nachfolger. Die letzte Nachricht stammt vom 22. April 1608 und erwähnt die zuletzt ergangene Mahnung. Dann bricht die Akte ab, sodass wir nicht erfahren, wie es letztlich ausging.

Soviel dürfte aber sicher sein, dass sich die finanzielle Situation der Ruf(f)ina und des Wolf Heinrich von Ega nicht grundlegend verändert hatte. Nach dem Tod des Georg Wolf von Hardheim stellten dessen Eigenerben Forderungen über 100 geliehene Reichstaler, ${ }^{89}$ für die seinerzeit Frau Gertraud von Rechberg zu Hohenrechberg geborene Burgmilchling gebürgt, sowie über weitere 50 Reichstaler, die sie Ruf(f)ina geliehen hatte. Die Eigenerben brachten die Sache vor das Rottweiler Hofgericht, das auch in ihrem Sinn entschied.

Der nächste und letzte bekannte Kreditgeber war der Ritterkanton Odenwald, aus dessen Kasse Wolf Heinrich von Ega $400 \mathrm{fl}$ geliehen hatte. ${ }^{90}$ Bürgen des am 22. Februar 1616 geschlossenen Vertrags waren Wolf Christoph von Gebsattel zu Uffenau und Lobenau, Hans Philipp Hund von Wenkheim und Wolf Reinhard von Stettenberg zu Gamburg. Auch hier kennt man den Ausgang nicht.

\section{Wolf Heinrich von Ega und der Kirchenpatronat}

Die Nennung der kirchlichen Gerechtsame leitet zum nächsten Gesichtspunkt über. Hinter dem Begriff Patronat verbirgt sich im Schüpfergrund ein höchst kompliziertes Gebilde. ${ }^{91}$ Der Patronat beinhaltete zunächst nur das Ius praesentandi, wobei sich dann folgender Nexus herausbildete, dass Hohenlohe dem betreffenden Pfarrkandidaten gleichsam automatisch die Bestätigung erteilte. Hier kamen nun die beiden anderen Ganerben Rosenberg und Stetten zu Kocherstetten ins

89 StAL B 94a Familienarchiv von Hartheim, Bü 2: Inventarium, fol. 140v-141r, 149r, 188r.

90 StAL B 583 U 5.

91 Helmut Neumaier: Iura episcopalia evangelischer Reichsritter? - Die Ganerbschaft Schüpf als Fallstudie. In: Jahrbuch für badische Kirchen- und Religionsgeschichte 7 (2013), S. 232-252. 
Spiel, wobei zweifellos die Brüder Georg Sigmund und Albrecht Christoph von Rosenberg, deren Dominanz innerhalb der Ganerbschaft unübersehbar ist, als die treibende Kraft anzusehen sind. Sie bestritten keineswegs die kirchlichen Rechte der Dienheim, wollten sie aber lediglich auf die Pfarrpräsentation beschränkt sehen. Im Sinne der von evangelischen Kirchenjuristen formulierten Episcopallehre beanspruchten die Rosenberger als Mitdorfherren Anteil an der eigentlichen Kirchenherrschaft. Dieses Jus episcopale sollte allerdings von allen drei Ganerben gemeinsam als - wie es viel später hieß - Episcopus ausgeübt werden. ${ }^{92}$

Im Jahre 1613 war in Schüpf die Pfarrbesetzung wieder akut geworden. ${ }^{93}$ Ega zeigte sich keineswegs gewillt, die Einschränkung der Dienheimschen Rechte hinzunehmen. In Art eines fait accompli unternahm er den Versuch, vollendete Tatsachen zu schaffen, indem er einen eigenen Pfarrkandidaten nominirte, um ihn am folgenden Tag examinieren und auf die Cantzel stellen $\mathrm{zu}$ lassen. Offenbar hatte Albrecht Christoph von Rosenberg von dieser Absicht erfahren, erschien in Schüpf und machte Egas Alleingang zunichte. Dessen Beschwerden beim Lehnherrn und beim Gesamtausschuss der sechs Orte der fränkischen Reichsritterschaft blieben wirkungslos. Die beiden Brüder von Rosenberg wiesen die Ansprüche Egas zurück, es gebühre denen von Oberschüpf durchaus nicht, aufgrund ihrer Collatur, d. h. des Patronatsrechts das Ius examinandi, instituendi und destituendi zu beanspruchen, sich vielmehr auf das Ius nominandi, vocandi und praesentandi ad Examen zu beschränken. Wolf Heinrich von Ega wie auch Stetten akzeptierten den im Grunde von den Rosenberg oktroyierten Rechtsnexus. Das schien ihnen allein schon deshalb geboten, weil mit dem Jahre 1617 ja das Centenarium der Reformation bevorstand und Eintracht das oberste Gebot sein musste. Die drei Wappen an der Kanzel sind sowohl Ausdruck der gemeinsamen Kirchenherrschaft als auch einer (fragilen?) Einigkeit.

Zunächst lässt sich ein modus vivendi bei der Besetzung der Kirchenstellen erkennen. Leutwein überliefert den Text des Präsentationsschreibens vom 23. Januar 1622 für den Nachfolger des verstorbenen Diakons M. Wolfgang Clemens Maier. ${ }^{94}$ Dieses Dokument ist die erste und für lange Zeit das letzte Archivale zur Besetzung einer Kirchenstelle im Schüpfergrund, weshalb es im Wortlaut wiedergegeben wird:

Woledle, Gestrenge, denselben seye unser freundliche willige Dienst samt Wünschung von dem Allmächtigen eines glückseeligen, friedlichen und freudenreichen Jahrs zuvor. Insonders freundtliche, liebe Vettern, Schwager, respe Gevatter und vertraudter Bruder.

92 Jakob Ernst Leutwein: Schüpfer Kirchenhistorie. Des zweyten Theils der Schüpfer Historie. Drittes Buch, Cap. IV, S. 7 f.; Iura episcopalia (wie Anm. 91), S. 245 f.

93 Ders.: Kirchenhistorie [...] Drittes Buch, Cap. Kirchenhistorie, Cap. IV S. 7 f.

94 Ebd., S. 14. 
Ihnen wird zweifelsohne nunmehr auch unverborgen seyn, daß der Allmächtige Gott nach seinem allein weisen Rath und vätterlichem Willen unlängsten weyl. den würdigen und wolgelehrten M. Wolf Clemens Maier, geweßenen Diaconum zu Schüpf, nunmehro seeligen, durch den zeitl. Tod von dieser Welt ab und ohnzweifentl. zu sich in die ewige Freud und Seeligkeit erfordert, wodurch dann die Caplaney erledigt worden, anjetzo aber die Presentation zu Ersetzung solcher Stelle an uns verhiret (?), alß ist nach gehaltener fleißiger Nachfrag des Briefszeiger, Wilhelm(us) Binz von Schwäbisch Hall, Studiosus Theologiae, com(m)endiret und gerühmet worden, derentweg(en) an die Vettern, Schwäger und Gevattern wir erstbenan(n)ten Studiosum Herkom(m)en gemäß, auch solcher Gestalt abfertigen und presentiren wollen, daß, woferne es ihnen gleichmäßig beliebig, er auf das fürderlichste sich in Predigen solle vernehmen laßen und darauf gebührlichen examiniret und, sofern er genugsam qualificiret erfunden, hernacher ordiniret und installiret werde. Da er aber in der Predigt und Examine wider verhofen nicht genugsam qualificiret befunden würde, sind wir erbietig, alsobalden nach einer andern Person zu trachten. Und wo es den Vettern, Schwägern und Gevattern gleichfalß beliebbig, könnte mehr gemelter Studiosus also balden an den Herrn Superintendenten gewiesen und eines gewißen Tags, an welchem die Probpredigt und Examen vorgehen solte, verglichen werden. Wie dann Ihrem Belieben nach Sie entweder in der Person oder durch Ihre Abgeordnete dem Actu beywohnen mögen, damit erheischender Nothdurft nach zu Beförderung des Gottesdiensts die Caplaney widerum ersetzt und deßwegen niemand sich zu beschwehren haben möge. So den Vettern, Schwägern und Gevatter wie Gestalt der Sachen nach freundlich unverhalten laßen wollen, damit allerseits göttl. Obacht treulichst befehlend. Datum d(en) 23sten Januarii An(n)o 1622.

\section{Eberhard von Stetten \\ Wolf Heinrich von Ega}

Dieses Präsentationsschreiben leiteten Ega und Eberhard von Stetten den Brüdern Albrecht Sigmund und Georg Christoph von Rosenberg zu. Der Kandidat Binz erhielt jedoch nicht das Plazet der Rosenberg. Leutwein kommentierte, er konnte über den Grund nichts in Erfahrung bringen. Vermutlich hatten die Rosenberg einen eigenen Kandidaten vorgesehen, den sie gegen Ega und den Stetten durchzusetzen vermochten. Binz wurde Ludimoderator (Schulmeister) in Lendsiedel, danach Pfarrer in Enslingen. ${ }^{95}$

Neben den Besetzungsstreitigkeiten um die Pfarrei Schüpf agierte Ega auch im Zusammenhang der Pfarrei Uiffingen. Ritter Albrecht von Rosenberg hatte hier die Reformation eingeführt, was ihm deshalb gelang, weil ihm das Neumünsterstift zu Würzburg die Präsentation zugestanden hatte. In der Folgezeit bildete

95 Otto Haug (Bearb,): Baden-Württembergisches Pfarrerbuch. Bd. II/2: Pfarrerbuch Württembergisch Franken. Stuttgart 1981, S. 225. 
sich ein Verfahren heraus, das Neumünster zunächst mehr als widerwillig akzeptierte, das im 18. Jahrhundert zum Automatismus wurde. Die Uiffinger Pfarrbesetzung war deshalb für die Ganerben so wichtig, weil der Uiffinger Pfarrer gleichzeitig Superintendent der gesamten Rosenbergischen Geistlichkeit war. Anscheinend lehnte das Stift mehrmals die Bestätigung ab, woraus sich die paradoxe Situation ergab, dass der Superintendent gleichsam illegal amtierte. Nach mehreren gescheiterten Anläufen präsentierten die Ganerben Georg Sigmund und Albrecht Christoph von Rosenberg, Wolf von Stetten zu Kocherstetten und Wolf Heinrich von Ega am 28. Mai 1614 dem Stiftspropst Erhard von Lichtenstein den schon einige Jahre in Uiffingen amtierenden Superintendenten M. Erhard Happach als Uiffinger Pfarrer. ${ }^{96}$ Diesmal hat das Stift akzeptiert.

\section{Wolf Heinrich von Ega und seine Nachkommen}

Ruf(f)ina von Leyen verwitwete Dienheim verheiratete Ega starb am 20. April 1623 (a. St.). Wann ihr Wolf Heinrich im Tod nachfolgte, konnte auch Leutwein nicht ermitteln. ${ }^{97}$ Legt man die Steuerverzeichnisse des Orts Odenwald der Jahre 1630 und 1640 zugrunde, ${ }^{98}$ erscheint der Name Ega schon im ersteren nicht mehr. Man wird davon ausgehen dürfen, dass das Schriftgut Wolf Heinrichs von Ega an die Erben überging. Da auch diese nicht lange - wie Leutwein sagte - florirten, ist es wohl vernichtet. Dokumente zu seiner Person finden sich ausnahmslos in fremden Archiven. Um dem negativen Befund noch einen weiteren anzufügen, ist das Fehlen eines Epitaphs oder auch nur einer Grabplatte zu konstatieren. Diese Aussage gilt ausnahmslos für alle Erben des Ritters Albrecht von Rosenberg. Umso dankbarer hat man Leutwein zu sein, dass bei allen urkundlichen Lücken wenigstens eine knappe narrative Quelle ein Schlaglicht auf Ega werfen lässt. Ohne seine ,Epitomae Schupfensis' hätte man schwerlich den Blick auf Vorarlberg und die Grafen von Hohenems gerichtet, ja wären sogar die Schüpfer Verwandtschaftsbeziehungen im Dunkeln, Wolf Heinrich von Ega wäre nur ein Name geblieben.

Neben dem im See ertrunkenen Wolf Georg ist ein weiterer Sohn der Ruf(f)ina aus der zweiten Ehe bezeugt. Dieser Philipp Ludwig suchte sein Glück in Kriegsdiensten. ${ }^{99}$ Schon 1620 ist er bei Worms in der ligistischen Armee nachzuweisen, um dann als Rittmeister im bayerischen Kürassierregiment des Oberst Andreas

96 Helmut Neumaier: Das Simultaneum in Uiffingen. Zur Interpretation des Normaljahres 1624. In: Würzburger Diözesangeschichtsblätter 67 (2005), S. 193-204, hier S. 197; Leutwein: Geschichte (wie Anm. 9), S. 165.

97 Leutwein (wie Anm. 9), S. 165.

98 StAL B 583 Bü 192, fol. 101-102 u. 99.

99 Leutwein (wie Anm. 9), S. 165 f.; auf Leutwein fußend Carl Wilhelm Friedrich Ludwig Stocker: Der Schüpfergrund und seine Besitzer. In: Freiburger Diözesanarchiv 25 (1896), S. 151-193, hier S. $186 \mathrm{f}$. 
Kolb von Rheindorff zu avancieren. Auf dem Marsch nach Tübingen heiratete er laut eines Heiratsbriefes, den Leutwein noch einsehen konnte, am 17. März 1635 Barbara Sibylla Truchseß von Höfingen aus schwäbischer Reichsritterfamilie (bei Leonberg). 1638 erlangte er die Beförderung zum Obristleutnant im genannten Regiment. In eben diesem Jahre blieb er, wie Leutwein sagt, d.h. er starb wohl den Soldatentod. Mit dem Sohn des Wolf Heinrich, dessen Biographie so viele ungeklärte Fragen aufwirft, endeten die von Ega im Mannesstamm. ${ }^{100}$

Aus der Ehe des Philipp Ludwig von Ega mit Barbara Sibylla Truchseß gingen zwei Töchter hervor, die unter Vormundschaft des Burkhard Dietrich von Weiler und eines Rittmeisters von Auerbach standen. Die ältere, Maria Philippina, die - wie Leutwein es ausdrückte - etwas Blöde im Gesicht gewesen, also an Sehschwäche litt, trat im Alter von 30 Jahren in das im Thurgau gelegene Kloster Diesenhofen ein. Ihre Schwester Anna Veronika wurde einem Tübinger Buchbinder in Kost gegeben. Sie heiratete dann als Alleinerbin der Oberschüpfer Besitzungen im Jahre 1661 den Wolf Adolf von Butlar zu Lobenbach. Nach Kriegsdienst wurde dieser Bambergischer Amtmann zu Weissenau, fiel in der Schlacht von St. Gotthard an der Raab und hinterließ ein Töchterchen Anna Eva, aber auch beträchtliche Schulden.

Die Witwe heiratete im Jahre 1666 den Ellwangischen Oberjägermeister und Oberamtmann zu Dannenberg Johann Jakob von Bernhausen (1630-15. Juni 1700), der damit Anteil am Schüpfergrund erhielt. Sie selbst starb am 12. September 1672 und wurde nebst vier Kindern im Chor der Kirche von Kupprichhausen bestattet, doch haben sich keine Grabplatten oder Epitaphien erhalten. Dieser Begräbnisort lässt erkennen, dass die weiblichen Nachkommen des Philipp Ludwig von Ega katholisch geworden waren. So verwundert es auch nicht, dass sich in St. Burkhard zu Würzburg die Memoria der Anna Veronika findet. Im Grabdenkmal des Johann Philipp von Hoheneck (1687-27. August 1743) zeigt der zweitunterste Schild der Ahnenprobe das Egasche Wappen. ${ }^{101}$ Sie war die Großmutter mütterlicherseits des Hoheneck.

Zwei Generationen der Familie von Ega im Schüpfergrund, die gleichsam kometengleich hier auftauchte und spurlos verschwand. Für sie gilt aber das Wort des Soziologen Pierre Bourdieu: „Durch den Eigennamen [...] wird eine gleichbleibende und dauerhafte soziale Identität gesetzt, die für die Identität des biologischen Individuums in all den möglichen Feldern einsteht, in die es als Akteur hineingerät". ${ }^{102}$

100 Andererseits werden im Memoriale von 1651 Stetten und Ega als Mitganerben genannt; vgl. Anm. 61.

101 Felix Mader (Bearb.): Die Kunstdenkmäler des Königreichs Bayern. Bd. III/12: Stadt Würzburg. München 1915, S. 162, Nr. 11.

102 Pierre Bourdieu: Praktische Vernunft. Zur Theorie des Handelns (edition suhrkamp, N.F. 985), Frankfurt 1998, S. 78 f. 